\title{
INDUSTRIAL INSURANCE
}

\author{
VI \\ PRIVATE INSURANCE COMPANIES \\ CHARLES RICHMOND HENDERSON \\ University of Chicago
}

\section{INDUSTRIAL LIFE INSURANCE}

The purpose of these companies, from the standpoint of directors and stockholders, is profit; their social end is to secure for the policy-holders a certain sum to provide for the expenses of mortal illness and for burial without appeal to charity. Some of these same corporations carry on an ordinary life-insurance business which does not in any important factor differ from other life-insurance agencies, and does not require special attention in this article. ${ }^{1}$

The vast importance and extent of the business of these burial insurance companies may be indicated by their statistics. In a previous part of this discussion the principal facts have been cited. The face promise of all policies of industrial companies in the year 1902 was $\$ 1,806,890,864$. The number of policies was I 3,448, I24, and the average value of the policies was $\$$ I $35 . \mathrm{Mr}$. Dryden estimated that the companies distributed annually to their beneficiaries more than $\$ 20,000,000$ in burial benefits.

The burden of this enormous business is heavy and is borne

${ }^{1}$ References: Frederick L. Hoffman, History of the Prudential Insurance Company of America, I875-19oo; Handbook and Rerference Guide to the Exhibits of the Prudential Insurance Company of America, prepared for the Louisiana Purchase Exposition, St. Louis, I904; John F. Dryden, The Inception and Early Problems of Industrial Insurance, I905; Description of Ordinary Policies of the various companies; article on "Industral Insurance," Encyclopedia Americana, by Haley Fiske, vice-president of the Metropolitan Life Insurance Company; Haley Fiske, Testimony before the Legislative Investigating Committee of New York, I905; H. Fiske, "Profits of Industrial Insurance," United States Review, thirtieth anniversary number; H. Fiske, "Industrial Insurance," Charities Review, March, I898; Memorandum submitted on behalf of the Metropolitan Insurance Company, respecting the proposed insurance bills, New York, 1906. 
exclusively by members of the wage-earning groups, and especially by those whose wages are lowest or next to the lowest. This expense has come to be regarded in this country as a necessary part of the weekly budget. There prevails among the people of our cities, among immigrants as well as among native born, a strong feeling against "pauper burials," and this sentiment is quickened and stimulated by the persuasions and representations of the numerous agents of the industrial insurance companies; it is their stock in trade. According to Hoffman (History of the Prudential Insurance Co., p. 289) the average policy in that company in I899 was for \$II4.22. The entire payments of premiums into the treasury of this company in I899 amounted to the sum of $\$ 19,028,792$, and the payments of benefits to $\$ 5,426,545$. The entire receipts from the beginning to the year I899 were $\$ \mathrm{r} 20,505,542$, and the payments of burial benefits $\$ 39,901,006$. The ratio of cost of administration to income was 39. I7 per cent., as compared with I7.34 per cent. in the ordinary life-insurance companies. At first sight this contrast is so startling, and the difference of cost so great as to raise a suspicion of foul play. But further analysis mitigates the severity of judgment, although it may lead us to dislike the system even more than before. (The figures may be found in the Standard of September I7, I898, pp. 314 ff., as given by Mr. J. R. Hegemann, president of the Metropolitan Life Insurance Company.) In Statistics, Fraternal Societies, 1905, p. $2 \mathrm{r} 3$, the editor asserts that in twenty-five of the ordinary lifeinsurance companies the ratio of administrative expenses to premiums was I8.3 per cent. (varying from ro.4 to $3 \mathrm{I} .7$ per cent.) ; while in twenty-five fraternal societies the cost was on the average only 8.4 per cent.

The explanation of the difference and of the enormous burden which falls upon the poor insurers is given by the administrators of the industrial companies themselves, at least in part. Of the cost for salaries and the amounts absorbed by profits of directors and stockholders we must learn elsewhere, but of the chief facts they make clear disclosure. One of the factors in explanation is the small size of the poor man's policy, as compared with that of the rich man. The average policy in ordinary companies is 
$\$ 2,468$, while that in industrial companies is only $\$ 142$, and that of the weekly payment plan is much lower still. The industrial company must write at least eighteen policies to make the sum of one policy in ordinary insurance. In connection with each of these little policies visits must be made to solicit and write the policies; each policy must be carefully examined by experts, immense correspondence must be carried on from the central office with agents all over the land, the payments of premiums demand time and expense, the accounts must be kept with each policy holder and each agent, and the medical examinations also call for heavy payments. It is estimated that the agents of these companies must make in the United States annually more than $4 \mathrm{I} 6,000,000$ visits in homes, or about $1,328,000$ each week day. To these causes of expense we must in fairness add the fact that the rate of mortality among working people is much higher than among the members of the well-fed, comfortable classes, and this makes the cost of insurance higher. The habits of life of many working-people, their unsanitary homes, inadequate or improper food, hard and monotonous labor often at depressing tasks, close confinement, and occasionally inherited defects, all have a bearing on death rates and hence on premiums which must be charged to cover risks. The table shows the relative rates of mortality according to Farr's English Life Table, based on the general population of Great Britain; the Actuaries' Table, based on the combined experience of seventeen English companies; and the table based on the experience of $12,000,000$ insured lives with the Metropolitan Life Insurance Company.

\begin{tabular}{|c|c|c|c|}
\hline Age & $\begin{array}{c}\text { Deaths per } \mathrm{I}, \infty 00 \\
\text { (Farr) }\end{array}$ & Actuaries & Metropolitan \\
\hline $\begin{array}{l}20 \ldots \ldots \\
21 \ldots \ldots \\
25 \ldots \ldots \\
35 \ldots \ldots \\
45 \ldots \ldots \\
55 \ldots \ldots \\
65 \ldots \ldots \\
70 \ldots \ldots\end{array}$ & $\begin{array}{r}7.74 \\
8.46 \\
9.24 \\
\text { II.24 } \\
14.50 \\
21.75 \\
41.20 \\
60.80\end{array}$ & $\begin{array}{r}7 \cdot 25 \\
7 \cdot 33 \\
7 \cdot 72 \\
9.19 \\
11.95 \\
20.99 \\
42.45 \\
62 \cdot 51\end{array}$ & $\begin{array}{l}10.5^{2} \\
\text { II.56 } \\
\text { I4.14 } \\
17.15 \\
22.5^{6} \\
35.22 \\
64.51 \\
90.99\end{array}$ \\
\hline
\end{tabular}

The actual premiums paid in the industrial companies are set forth in their tables, and typical tables are here reproduced. 
INDUSTRIAL INSURANCE OF THE INSURANCE COMPANIES

(Industrial Life Insurance, Its History, Statistics, and Plans [1905], pp. 59 ff.)

BALTIMORE LIFE, BALTIMORE, MD.

\begin{tabular}{|c|c|c|c|c|c|}
\hline \multirow{2}{*}{$Y_{E A R}$} & \multirow{2}{*}{$\begin{array}{l}\text { New Policies } \\
\text { IN This Year }\end{array}$} & \multicolumn{2}{|c|}{ Policies in Force } & \multirow{2}{*}{$\begin{array}{l}\text { Premiums } \\
\text { Received }\end{array}$} & \multirow{2}{*}{ Payments } \\
\hline & & Number & Amount & & \\
\hline r904 ...... & $\$ 4,548,840$ & 103,965 & $\$ 7,977,954$ & $\$ 592,777$ & $\$ 108,545$ \\
\hline I903 ...... & 4,969, I 52 & 105,587 & $7,770,477$ & 596,472 & $96,73^{1}$ \\
\hline I902 $\ldots \ldots \ldots$ & 5,723, I I8 & 100,292 & $7,133,760$ & 565,87 I & 7 I, 345 \\
\hline I9or ...... & $5,774, \circ 38$ & 100,7 I4 & $6,529,913$ & 544,309 & 68,429 \\
\hline I900 . . . . . & $5,354, \mathrm{r} 40$ & 92,3 IO & $5,642,853$ & 523,785 & 54,92 I \\
\hline I899 . . . & $4,486,2$ I 2 & $86,06 \mathrm{I}$ & $5, \circ 38,963$ & 467,880 & 47,599 \\
\hline I898 ........ & $4,45^{\mathrm{I}}, 355$ & $7^{6,974}$ & $4,25 \mathrm{I}, 9 \circ 5$ & 421,496 & 39,879 \\
\hline
\end{tabular}

Colonial, Jersey City, N. J.

\begin{tabular}{|c|c|c|c|c|c|}
\hline I9O4 $\ldots$ & $\$ 8,784,984$ & 83,690 & $\$ 9,78$ I, $3^{\text {I } 7}$ & $\$ 454,8 \circ 7$ & $\$ 1$ I 5,554 \\
\hline r9o3 ....... & $7,122,800$ & 70,076 & $8,028,103$ & 364,588 & 86,855 \\
\hline r902 . . & $6,454,644$ & 55,597 & $6,149,4$ IO & 282,093 & 69,87 I \\
\hline I90I ... & $5,2 \mathrm{I}, 3 \circ \mathrm{I}$ & 43,520 & $4,668,7^{6} 3$ & $2 \circ 3,454$ & 50,676 \\
\hline I900 ... & $4,209,047$ & 34,674 & $3,650,629$ & I 52,501 & 39,5 ㅇ \\
\hline I8́99 . . . . . . & $4,042,526$ & 27,697 & $2,854, \circ 75$ & 85,252 & I 5,900 \\
\hline I898 . . . . . & 2,7 I9,798 & 10,623 & $I, 423,482$ & 23,626 & $4,22 \mathrm{I}$ \\
\hline
\end{tabular}

Columbian National, Boston

\begin{tabular}{r|r|r|r|r|l}
\hline r904 $\ldots \ldots \ldots$ & $\$ 9,468,2$ I6 & 40,397 & $\$ 5,532,978$ & - & - \\
r903 $\ldots \ldots \ldots$ & 4,8 I3,94I & I8,756 & $2,630,959$ & - \\
r902 $\ldots \ldots \ldots$ & 759,640 & 4,275 & 6 I5,3 16 & - & - \\
\hline
\end{tabular}

Contentnea Life, Wilson, N. C.

\begin{tabular}{l|l|l|l|l|l}
\hline $\mathrm{r} 903 \ldots \ldots$ & $\$ 43,980$ & 798 & $\$ 22,360$ & $\$ 1,283$ & $\$ 5$ \\
\hline
\end{tabular}

Equitable Industrial, Washington, D. C.

\begin{tabular}{|c|c|c|c|c|c|}
\hline rgo4 $\ldots \ldots \ldots$ & $\$ 1,690,73^{6}$ & 25,522 & $\$ 2,960,2$ I 2 & $\$ 107,35^{8}$ & $\$ 34,599$ \\
\hline I903 ....... & $\mathrm{I}, 66_{5}, 4 \mathrm{I} 4$ & 23,304 & 2,692,699 & 95,425 & $24,40 I$ \\
\hline I9O2 ...... & I,334,923 & $20,40 I$ & $2,325,483$ & $58,25^{2}$ & I6,458 \\
\hline
\end{tabular}

Home, Wirmington, DeL.

\begin{tabular}{|c|c|c|c|c|c|}
\hline r9o3 ... & $\$ 284,400$ & I 2,948 & $\$ 1,691,809$ & $\$ 49,043$ & $\$ 14,954$ \\
\hline r902 ....... & $\mathrm{r}, \mathrm{I04}, 223$ & I 2,842 & $\mathrm{I}, 448,963$ & 48,487 & I 5,867 \\
\hline rgor ....... & I, $4 \mathrm{I} 7,440$ & I 2,132 & I,450,393 & $27,77^{\circ}$ & 8,633 \\
\hline r900 $\ldots \ldots \ldots$ & $\mathrm{x}, 55^{\mathrm{I}, 079}$ & 5,146 & 920,980 & $\mathrm{I} 8,5^{2} 3$ & 6,063 \\
\hline
\end{tabular}

ImMediate Benefit, Baltimore, MD.

\begin{tabular}{|c|c|c|c|c|c|}
\hline r904 . . . . . & $\$ 1,233,420$ & $I_{5}, 002$ & $\$ 1,2$ I0,675 & $\$ 7 I, 432$ & $\$ 18,086$ \\
\hline I903 ...... & $39 \mathrm{I}, 268$ & 10,703 & 705, I 28 & 58,857 & I6,245 \\
\hline r902 ........ & $42 \mathrm{I}, 3 \mathrm{I} 8$ & 7,587 & 509, I 31 & 54,996 & 14,253 \\
\hline rgor ....... & $44 I, 309$ & 7,201 & 406,849 & 44,760 & $\mathrm{I} 4,3 \mathrm{I} 4$ \\
\hline
\end{tabular}

This content downloaded from 080.082.077.083 on January 18, 2018 09:32:06 AM All use subject to University of Chicago Press Terms and Conditions (http://www.journals.uchicago.edu/t-and-c). 
INDUSTRIAL INSURANCE OF THE INSURANCE COMPANIES-Continued Immediate Benefit, BaltrmoRe, Md.-Continued

\begin{tabular}{|c|c|c|c|c|c|}
\hline \multirow{2}{*}{ YEAR } & \multirow{2}{*}{$\begin{array}{l}\text { New Policies } \\
\text { IN This Year }\end{array}$} & \multicolumn{2}{|c|}{ Policies IN Force } & \multirow{2}{*}{$\begin{array}{l}\text { PREMiUms } \\
\text { ReCEIVED }\end{array}$} & \multirow{2}{*}{ Payments } \\
\hline & & Number & Amount & & \\
\hline $\begin{array}{l}\text { r9oo } \ldots \ldots \ldots \\
\text { r899 } \ldots \ldots \ldots \\
\text { r898 } \ldots \ldots \ldots \\
\text { r897 } \ldots \ldots \ldots\end{array}$ & $\begin{array}{r}\text { I39,535 } \\
\text { I, IO2,6I8 } \\
\text { I,488,774 } \\
\text { I, } 520,337\end{array}$ & $\begin{array}{r}5, I 3 I \\
4,48 I \\
I 3,65 I \\
I I, 843\end{array}$ & $\begin{array}{r}228,5 \text { I } 2 \\
195,802 \\
\text { r, 006,704 } \\
845,954\end{array}$ & $\begin{array}{r}\$ 43,763 \\
43,693 \\
62,483 \\
38,282\end{array}$ & $\begin{array}{r}\$ I 4,004 \\
\text { I I,24I } \\
\text { I9,99I } \\
\text { II }, 073\end{array}$ \\
\hline
\end{tabular}

JoHn Hancock, Boston, Mass.

\begin{tabular}{|c|c|c|c|c|c|}
\hline r9o4 ....... & $\$ 6 r, 840,600$ & I,474,399 & $\$ 233,069,767$ & $\$ x_{3}, 622,35^{\circ}$ & $\$ 3,971,330$ \\
\hline r9o3 ....... & $57,444,640$ & I,395,779 & $2 \mathrm{I} 6,375,960$ & I $2,389,529$ & $3,642,68 \mathrm{I}$ \\
\hline r902 ...... & $68,137,409$ & $r, 3$ I 2,630 & $200,294,696$ & Io,9I 4,984 & $2,964,953$ \\
\hline I90I ....... & $57,928,75^{\mathrm{I}}$ & $I, 223,500$ & I77,597,439 & $9,595,301$ & $2,8 \mathrm{I} 9,624$ \\
\hline r900 ...... & $52,060,760$ & I,I 52,444 & I59,893,856 & $8,252,34 I$ & $2,554,9 \circ 5$ \\
\hline r899 . . . . . . : & $44,358,633$ & r,o69, I97 & I 4 I,609,904 & $7,209,290$ & $2,193,573$ \\
\hline r898 . . . . . . & $37,936,626$ & 956,382 & $124,923,200$ & $6,512,804$ & I, 874, OI 5 \\
\hline I897 ....... & 35,959, I76 & 899,4 I 8 & I $5,75^{\circ}, 7 \circ 9$ & $5,773, \mathrm{I} 44$ & $\mathrm{I}, 684,027$ \\
\hline r896 ....... & 36,87 I,080 & $835,35 \mathrm{I}$ & $105,640,047$ & $5,217,207$ & $\mathrm{I}, 643,708$ \\
\hline r895 ....... & $41,9 \circ 5,652$ & 771,972 & $95,640,574$ & $4,638,040$ & $I, 428,92 I$ \\
\hline r894 ....... & $33,146,067$ & $68 \mathrm{I}, 802$ & $82,876,33^{8}$ & $3,862, \circ 56$ & I,2 I 3,607 \\
\hline r893 ...... & 34,57 I,979 & 607,150 & $73,043,678$ & $3,444,3$ I 3 & I, I I6, I 24 \\
\hline I892. & $29,3^{26,680}$ & 556,435 & 65,428, I 21 & 2,9 I 4,498 & $\mathrm{I}, 005,5 \circ 7$ \\
\hline I89I ... & $25,374,745$ & 476,612 & $54,5^{16,5^{14}}$ & $2,3^{8} 7,7$ OI & 932,488 \\
\hline r89o ... & 23,083, I 5 I & 402,147 & $45,772,7 \circ 9$ & $2,002,644$ & $81 \mathrm{r}, 688$ \\
\hline r889 . . . . . & I8, 239,650 & 320,264 & $3^{6}, 3^{6} 5,4 \mathrm{I} 9$ & I,6I6,585 & 596,5 10 \\
\hline r888 . . . . & I $5,953,123$ & 256,574 & $29,943, \circ 5^{2}$ & I,3 I7,374 & 473,843 \\
\hline r887 $\ldots \ldots$ & ${ }^{1} 3,334,392$ & 203,467 & $23,802,502$ & $\mathrm{I}, \circ 3 \mathrm{I}, 845$ & 357,002 \\
\hline I886 ... & $\mathrm{I}, 748, \mathrm{I}^{2}$ & $\mathrm{I}_{4} 8,85^{\circ}$ & I7,805,9 го & $8 \mathrm{I}_{5}, 75^{\circ}$ & 306,938 \\
\hline I885 $\ldots$ & $7, \circ 55,933$ & IO7,872 & I $2,600,935$ & $647, \mathrm{I} 43$ & 221,266 \\
\hline I884 $\ldots \ldots$ & $4,752,6 \mathrm{I} 3$ & 80,629 & $8,940,275$ & $547, \circ 58$ & 206,460 \\
\hline$r 883 \ldots \ldots$ & $4,085,489$ & 63,625 & $6,730,902$ & 472,242 & I75,438 \\
\hline r882 ...... & $3,7 \pm 8,9 \circ 2$ & 48,568 & $5,096,488$ & 4 I 5,537 & I94,053 \\
\hline r88r .... & 2,93 I,860 & 36,0 I 2 & $3,787,230$ & 346,887 & 161,606 \\
\hline r880 . . . . . . & $5,483,43 \mathrm{I}$ & 30,702 & 3 , I39,oI8 & $336, x 98$ & $I_{72,570}$ \\
\hline r879 $\ldots \ldots$ & $\mathrm{r}, 275,9 \mathrm{r} 8$ & 9,327 & $95^{I, \infty}, 00$ & 294,043 & $\mathrm{I} 62, \mathrm{I} 4 \mathrm{I}$ \\
\hline
\end{tabular}

Life Insurance Company of Virginia, Richmond, Va.

\begin{tabular}{|c|c|c|c|c|c|}
\hline r904 $\ldots \ldots \ldots$ & $\$ \mathrm{Ir}, 788,596$ & $35^{8,54}$ & $\$ 37,7$ ro,9oI & $\$ r, 680,4 I r$ & $\$ 5$ I2, IO5 \\
\hline r903 ....... & II,906,867 & $33^{1,45^{2}}$ & $34,5 \circ 3,483$ & $\mathrm{r}, 5 \circ 9,46_{3}$ & 434,204 \\
\hline rgo2 ....... & I $2,437,33^{8}$ & 302,839 & $30,3 \circ 3,8 \times 5$ & $\mathrm{x}, 33^{8,5}, \mathrm{x}$ & 392,997 \\
\hline rgor ....... & $10,785,037$ & 266,685 & $26,9 \circ 6,073$ & $\mathrm{I}, \mathrm{I}_{5} \mathrm{I}, 2 \mathrm{I} 3$ & 373,4 I9 \\
\hline r9oo ....... & 9, I02,6 16 & 237,283 & $23,239,844$ & $\mathrm{x}, 087,272$ & 370,429 \\
\hline r899 . . . . . . & $8,968,321$ & 219,679 & $20,246,656$ & 937,901 & 308,259 \\
\hline$r 898 \ldots \ldots$ & I $2,318,555$ & I94,95I & I8,373, II9 & 852,028 & $25^{2,025}$ \\
\hline r897 . . . . . & $9,2 \mathrm{I} 2,26 \mathrm{I}$ & I65,660 & $I_{5}, 264,25^{\circ}$ & $75^{2,215}$ & $2 I_{4}, 273$ \\
\hline r896 . . . & $10,61_{3}, 052$ & x49,672 & I3,778, I99 & $7 \pm 2,932$ & 222,987 \\
\hline r895 . . . & $9,256,579$ & II 6,8 I 4 & I I,०53,०39 & $59 I, 38 I$ & I62,663 \\
\hline r894 . . . . . & $8,140,705$ & 99,6 I8 & $9,647,7 \circ 7$ & 551,794 & 160,003 \\
\hline r893 . . . . . & $6,998,569$ & 75, 工 30 & $6,643,06 x$ & 546, I 5 I & I 54,529 \\
\hline I892 . . . . . & $6,272,217$ & 69,527 & $6,230,224$ & 475,520 & I 20,496 \\
\hline r89r . . . . . . & $4,940,820$ & $5 \mathrm{I}, 49 \mathrm{I}$ & $4,520,424$ & 395 ,rgr & $\mathrm{IO} 2,742$ \\
\hline
\end{tabular}

This content downloaded from 080.082.077.083 on January 18, 2018 09:32:06 AM All use subject to University of Chicago Press Terms and Conditions (http://www.journals.uchicago.edu/t-and-c). 
INDUSTRIAL INSURANCE OF THE INSURANCE COMPANIES-Continued Life Insurance Company of Virginia, Richmond, Va.-Continued

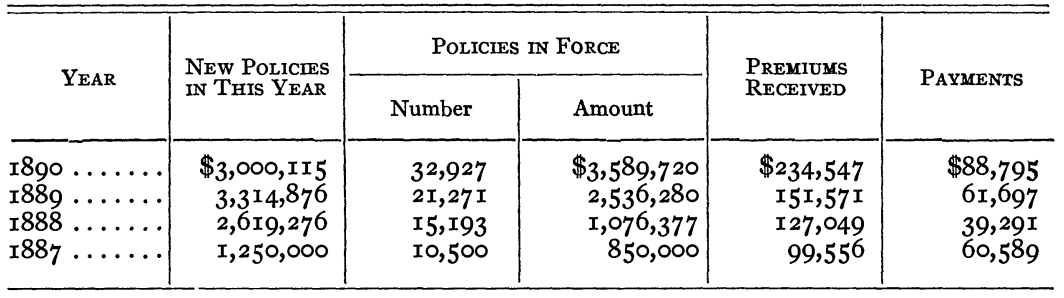

Metropolitan, New York, N. Y.

\begin{tabular}{|c|c|c|c|c|c|}
\hline I904 & $\$ 305,258,155$ & $7,614,729$ & $\$ 1,127,889,229$ & $\$ 50,808,924$ & $\$ 14,826,976$ \\
\hline I9०3 & $297,968,863$ & $7,187,345$ & $\mathrm{I}, 059,875,827$ & $45,656,96 \mathrm{I}$ & I $2,907,6 \mathrm{I} 7$ \\
\hline I902 & $3^{12,990,33^{8}}$ & $6,698,29 \mathrm{I}$ & $981,676,306$ & $39,653,725$ & II, 320,967 \\
\hline I901. & $296,606,312$ & $6,008,662$ & $88 \mathrm{I}, 49 \mathrm{I}, 45 \mathrm{I}$ & $34,7 \circ 5,186$ & I0,704,747 \\
\hline I900. & $264,737,682$ & $5,327,067$ & $768,977,676$ & $3 \mathrm{I}, 2 \mathrm{IO}, 356$ & $9,785,624$ \\
\hline I899. & $253,396,620$ & $4,855,75^{6}$ & 688,629, I 75 & $26,59 \mathrm{I}, 65 \mathrm{I}$ & 8,575, 工 34 \\
\hline I898 & $210,508,694$ & $4,3 \mathrm{I} 7,274$ & $591,427,272$ & $23,372,770$ & $7,691,943$ \\
\hline ז̊897 & $232,264,188$ & $4,028,722$ & $534,343,75^{6}$ & $21,402,966$ & $6,990,866$ \\
\hline I896. & I69,820,543 & $3,643,569$ & $454,068,004$ & I9,306, 196 & $6,963,25^{6}$ \\
\hline I895. & I $75,905,407$ & $3,45^{8,846}$ & $416,062,194$ & I8,336,918 & $6,580,390$ \\
\hline I894 & $294,270,45^{I}$ & 3,559, I65 & $423,5^{\mathrm{I} 4}, \mathrm{I} 7 \mathrm{I}$ & I6,827,0I6 & $5,747,823$ \\
\hline I893. & $150,057,7 \circ 3$ & $2,93^{2}, 064$ & $343,917,746$ & $\mathrm{I} 4,36 \mathrm{I}, 2 \mathrm{I} 4$ & $5,535,120$ \\
\hline I892. & I $27,222,470$ & $2,7 \simeq 5,414$ & $305,45^{\mathrm{I}}, 576$ & $\mathrm{I} 2,5 \mathrm{II}, 078$ & $4,898,3^{82}$ \\
\hline I89i. & $94,927,488$ & $2,278,487$ & $254,939,88 \mathrm{I}$ & 10,830,373 & $4,408,379$ \\
\hline I890. & $100,852,802$ & $2,096,595$ & $23 \mathrm{I}, \mathrm{II} 5,440$ & $9,390,927$ & $3,746,478$ \\
\hline I889. & $92,726,883$ & I, 849, I I 3 & $200,829,929$ & $8,342,945$ & $3,042,8 \mathrm{I} 8$ \\
\hline I888 & $91,242,946$ & $I, 632,642$ & $\mathrm{I} 76,533, \mathrm{I} 42$ & 6,810,1 10 & $2,550,105$ \\
\hline I887 & 84,059, I I 8 & $\mathrm{I}, 345, \mathrm{I} 25$ & $\Upsilon 47,758,287$ & $5,618,767$ & $2,098,936$ \\
\hline 886 & $72,783,721$ & I,066,875 & II $9,560,339$ & $4,438,096$ & $\mathrm{I}, 566,5^{\mathrm{I} 4}$ \\
\hline 1885 & 57,8 19,9 1 2 & 829,833 & $9 \mathrm{I}, 234,25^{2}$ & $3,414,524$ & I, 279,645 \\
\hline & $59,5 \circ 5,42 I$ & 670,999 & $7 \mathrm{I}, 965,635$ & $2,8 \mathrm{II}, 8 \mathrm{I} 6$ & $970,59^{\circ}$ \\
\hline I883 & $52,5 \circ 5,697$ & $5^{26,042}$ & $56,536,3^{25}$ & $\mathrm{I}, 975,703$ & 631,639 \\
\hline 1882 & $36,822,169$ & 335,789 & $34,679,307$ & I, $246,5^{I} 5$ & $3^{6} 9,3$ I4 \\
\hline I88I & $24,469,300$ & I90, 348 & I $7,894,620$ & 859,057 & $268,8 \mathrm{II}$ \\
\hline I880. & $20,728,700$ & IÍ,I93 & 9, IO3,870 & 568,204 & 200,805 \\
\hline I879 . & 523,539 & 5, I 43 & $5^{16,618}$ & $43^{2,560}$ & $\mathrm{I} 44,42 \mathrm{I}$ \\
\hline
\end{tabular}

Mutual of Baltimore, Baltimore, MD.

\begin{tabular}{|c|c|c|c|c|c|}
\hline I904 $\ldots \ldots \ldots$ & $\$ 2,016,9$ I 8 & $35,73^{\circ}$ & $\$ 3,559,495$ & $\$ 207,443$ & $\$ 84, \circ 75$ \\
\hline rg०3 $\ldots \ldots \ldots$ & I,8I 5,548 & 32,162 & $3,095,453$ & $\mathrm{I} 88, \mathrm{I} 58$ & 74,508 \\
\hline $1902 \ldots \ldots \ldots$ & $\mathrm{I}, 684,67 \mathrm{I}$ & 28,093 & $2,64 \mathrm{I}, 35^{6}$ & I65,322 & 57, II \\
\hline I90I ... & $\mathrm{I}, 322, \mathrm{II} 2$ & $23,98 \mathrm{I}$ & $2,206,08 \mathrm{I}$ & 147,363 & 59,097 \\
\hline $1900^{\circ} .$. & I, 217,455 & 20,855 & $\mathrm{I}, 872,030$ & I $28,20 I$ & 43,822 \\
\hline I8́99 . & 300,572 & 17,574 & $\mathrm{I}, 526,787$ & 124,502 & 52,243 \\
\hline I898 .. & $\mathrm{I}, 454,344$ & I9,OI5 & $\mathrm{I}, 479,364$ & II9,286 & 44,355 \\
\hline I897. & $\mathrm{I}, \mathrm{I} 48,486$ & I 4,746 & I, 049,638 & เог, 676 & $43,4 \mathrm{IO}$ \\
\hline 1896 . & $55^{\circ}$, oI 3 & 8, I $55_{5}$ & $62 \mathrm{I}, \mathrm{IO} 8$ & 93,47 I & 32,192 \\
\hline $1895 \ldots$ & 495,226 & 5,999 & $54 \mathrm{I}, 308$ & 85,493 & 34,423 \\
\hline I894 & 387,993 & 4,340 & 446,501 & 73,225 & $3^{1,792}$ \\
\hline I893. & 602,196 & 2,028 & 243 , O10 & $68,5^{6} 5$ & 25,643 \\
\hline I892. & 440,989 & $2,85^{6}$ & $391,85^{6}$ & 62,691 & 2I,493 \\
\hline
\end{tabular}


INDUSTRIAL INSURANCE OF THE INSURANCE COMPANIES-Continued Providence Life, Providence, R. I.

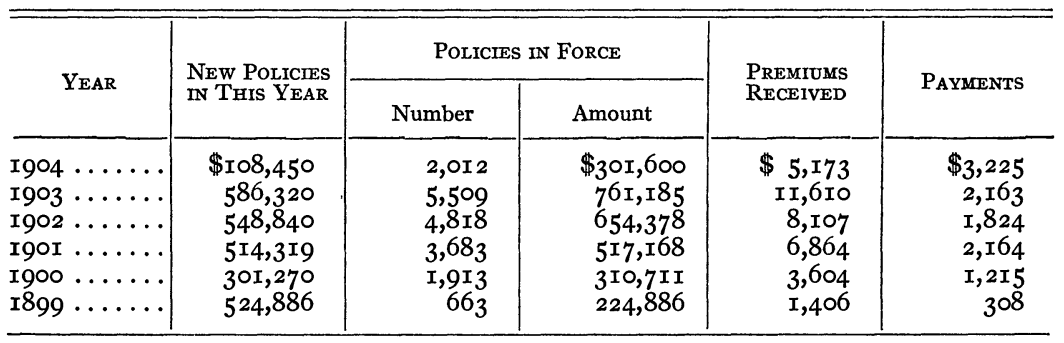

Prudential, Newark, N. J.

\begin{tabular}{|c|c|c|c|c|c|}
\hline 04 & $\$ 202,524,9$ II I & $5,642,335$ & $\$ 675,992,239$ & $\$ 4 \mathrm{I}, \mathrm{I} 55,697$ & $\$ \mathrm{II}, 258,506$ \\
\hline 903 & I90,386,294 & 5, 工 76,456 & 6r $3,935,9$ 工о & $36,028,402$ & $9,8 \div 2,45^{8}$ \\
\hline 1902 & $184,327,303$ & $4,692,182$ & $55^{\circ}, 464,265$ & $3 \mathrm{I}, \mathrm{I} 38,7 \mathrm{I} 8$ & $8,096,7$ I9 \\
\hline I9OI. & I9I, 7 I 2,877 & $4,290,539$ & 498, I $27, \mathrm{I} 33$ & $26,68 \mathrm{I}, 757$ & $7,411,428$ \\
\hline I900. & 0,423 & $5,908,622$ & $448,596,996$ & $22,559,354$ & $6,207,4 \mathrm{I} 8$ \\
\hline 899 . & $165,760,248$ & $3,406,189$ & $389,039,257$ & I9,028,792 & $5,420,758$ \\
\hline 898 & I $21,080,784$ & $2,924,526$ & $333,992,200$ & $16,139,452$ & $4,749,885$ \\
\hline 897. & I $12,37 \mathrm{I}, 379$ & $2,658,700$ & $303,770,95^{2}$ & $\mathrm{I} 4,55 \mathrm{I}, 868$ & $4,342,562$ \\
\hline 896 . & $108,223,7 \mathrm{I} 2$ & $2,437,25 \mathrm{I}$ & $279,030,63^{8}$ & I3,329,644 & $4, I 58,83^{I}$ \\
\hline 395 . & I $24,374,407$ & $2,33^{\circ}, 74 \mathrm{I}$ & $268,4 \mathrm{I} 4,100$ & II,892,766 & $3,846,754$ \\
\hline 894 . & $205, \mathrm{I} 28,243$ & $2,256,014$ & $259,840,927$ & I0,890,302 & 3,I9I,I 75 \\
\hline 393. & I $28,208,94 \mathrm{I}$ & $\mathrm{I}, 533$ & 99,566 & $9,084,844$ & $2,893,708$ \\
\hline 92. & $92,677,524$ & & 26,206 & 25,844 & $2,518,567$ \\
\hline 891 & $72,966,176$ & $1,360,3$ & I50,758,907 & & $2,079,669$ \\
\hline 890 & 74,484 & I, $228,33^{2}$ & I $35,084,498$ & $5,63^{6,876}$ & $\mathrm{I}, 749,7 \mathrm{I} 4$ \\
\hline 389 & 6,853 & $I, O S$ & II7 & & I, $327,85^{6}$ \\
\hline 888 & $4,98 \mathrm{I}$ & 850,064 & 3,854 & & I,096,234 \\
\hline & $60,202,194$ & & & & 853,819 \\
\hline 386 & 49,1 & & 59 & & 593,273 \\
\hline & 28, & $422,67 \mathrm{I}$ & 40, & & 418,622 \\
\hline 384 & $24,892,268$ & $3^{24,794}$ & & & $3^{22,382}$ \\
\hline & $20,426,140$ & 273 & & & 222,083 \\
\hline & II $, 54 \mathrm{I}, 2 \mathrm{IO}$ & 196,007 & I5,738,973 & 57 I,595 & I $57,7 \circ 5$ \\
\hline & $9,688,362$ & I $33,5^{82}$ & & & III, 508 \\
\hline & $8,555,904$ & 87,462 & $7,347,892$ & $250,95^{8}$ & $57,25^{6}$ \\
\hline 79 & $3,157,35^{2}$ & & & I 21,560 & 23, OI 3 \\
\hline & $\mathrm{x}, 785$ & & $2,027,888$ & 59,8 I 7 & II, $33^{8}$ \\
\hline & $967,93^{2}$ & II,226 & $\mathrm{I}, 030,655$ & $28,5 \mathrm{I} 7$ & 5,296 \\
\hline & 727, I68 & $4,8 \mathrm{I} 6$ & 443,072 & I 4,495 & $\mathrm{I}, 95^{8}$ \\
\hline
\end{tabular}

Western and Southern, Cincinnati, O.

\begin{tabular}{|c|c|c|c|c|c|}
\hline I904 $\ldots \ldots \ldots$ & $\$ I_{4}, 830$, I IO & 227,624 & $\$ 24,600,187$ & $\$ I, 249,945$ & $\$ 354,666$ \\
\hline r9o3 ...... & I4, I6r,533 & 209,623 & $22,223,035$ & I, I I3,699 & 295,369 \\
\hline I902 ....... & I $2,948,340$ & I84,686 & I9,643,480 & 9r7,337 & $22 \mathrm{I}, 284$ \\
\hline I90I ... & Io, 878,524 & I 55,096 & I6,426,534 & 722,605 & 203,896 \\
\hline I900 ... & I0,070,944 & I 3 I, I $3^{2}$ & г 3,6 I 8,878 & $6 I 4,30 I$ & I 50,642 \\
\hline I899 .... & $8,370,729$ & I 7,545 & го,88 I,96 г & 508,900 & I 22,494 \\
\hline I898 . . . & $7,256,666$ & 91,589 & $8,392,902$ & 397,687 & 96,448 \\
\hline r897 $\ldots \ldots \ldots$ & $4,980,043$ & $7 \mathrm{I}, 30 \mathrm{I}$ & $6,6 \mathrm{Ig}_{9}, 653$ & 320,996 & 79, I69 \\
\hline r896 . . . . . . . & $4,290,33^{2}$ & 62,747 & $5,724,728$ & 298,686 & 79,060 \\
\hline
\end{tabular}


INDUSTRIAL INSURANCE OF THE INSURANCE COMPANIES-Continued

$$
\text { Western and Southern, Cincinnati, O.-Continued }
$$

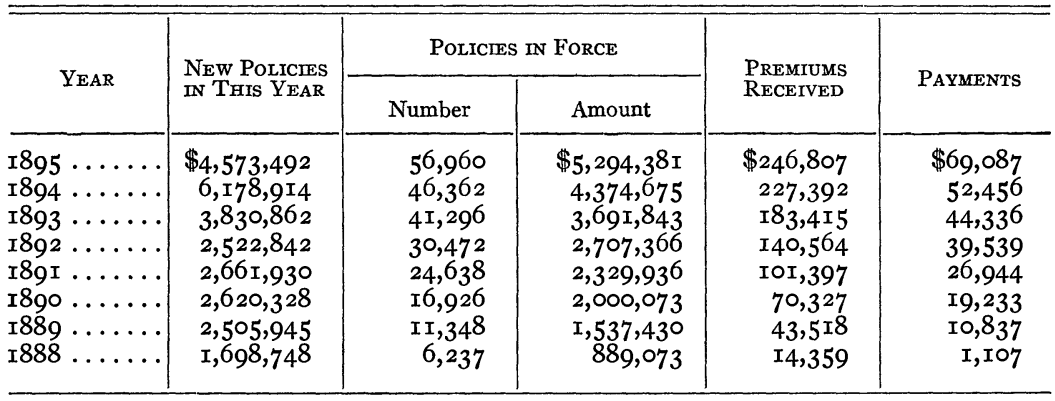

\section{GENERAL SUMMARY}

\begin{tabular}{|c|c|c|c|c|c|c|}
\hline \multirow{2}{*}{ YEAR } & \multirow{2}{*}{$\begin{array}{c}\text { No. } \\
\text { OF } \\
\text { CoM- } \\
\text { PANIES }\end{array}$} & \multirow{2}{*}{$\begin{array}{l}\text { NEW Policies } \\
\text { IN ThIS YEAR }\end{array}$} & \multicolumn{2}{|c|}{ Policies IN Force } & \multirow{2}{*}{$\begin{array}{l}\text { PREMIUMS } \\
\text { RECEIVED }\end{array}$} & \multirow{2}{*}{ Payments } \\
\hline & & & Number & Amount & & \\
\hline I904... & I4 & $\$ 624,422,3$ I6 & I $5,637,692$ & $\$ 2,132,300,723$ & $\$$ I Iо, , 6,643 & $\$ 31,302,626$ \\
\hline I9०3... & I 5 & 596,5 10,565 & $\mathrm{I} 4,600,502$ & $\mathrm{I}, 977, \mathrm{I} 85,534$ & $98,06_{3}, 490$ & 27,408 , I9I \\
\hline I902 ... & I5 & $610,968,8$ I9 & I $3,444,753$ & I, $806,454,74^{2}$ & 85, I 46,4 Iо & $23,243,657$ \\
\hline I901... & I5 & $.598,593,825$ & I $2,333,459$ & I, $640,398,546$ & $74,660,060$ & $22,003,402$ \\
\hline I900... & r8 & $566, \circ 37,936$ & I I, 2 I $5,53 \mathrm{I}$ & $\mathrm{I}, 468,474,534$ & $65,962,426$ & I9,607,808 \\
\hline I899... & I7 & $5^{19,796,08} 5$ & I0, 048,808 & $\mathrm{I}, 292,8 \mathrm{I} 2,402$ & 56, I 59,889 & I $7,0_{23}, 485$ \\
\hline I898... & I4 & $422, \mathrm{I} 64,8 \mathrm{ro}$ & 8,794, I7 8 & I, I09, 526,870 & $48,776,246$ & $\mathrm{r} 4,97 \mathrm{r}, 238$ \\
\hline I897... & I 2 & $4 \mathrm{I} 5,338,6 \mathrm{I}_{4}$ & $8,000,636$ & $995,545,73^{6}$ & 43,6 r9,3 & I $3,526,3$ I 5 \\
\hline I896... & II & $360,852,45^{8}$ & $7,375,688$ & $886,484,869$ & $40,058,7$ or & I $3,420,336$ \\
\hline I895 ... & I I & $380,832,362$ & $6,943,769$ & $8 \mathrm{I} 9,52 \mathrm{I}, 573$ & $37,008,536$ & I $2,398,782$ \\
\hline I894... & I2 & $573,279,943$ & $6,847,892$ & 802,016, I 33 & $32,253,88 \mathrm{I}$ & Io,635,602 \\
\hline I893... & II & $344,36 r, 223$ & 5,748, I95 & $66 \mathrm{r}, 568,502$ & 28,3 I, 386 & $9,955,865$ \\
\hline I892... & ro & $276,893,923$ & 5, I 18,897 & 582,7 10,309 & $24,352,900$ & $8,84 \mathrm{I}, 322$ \\
\hline I89 .... & 9 & 218, I 38,800 & $4,302,427$ & $481,060,7$ I6 & $20,654,980$ & $7,725,328$ \\
\hline I890... & 9 & $242,250,959$ & 3,875 , IO2 & $428,037,245$ & I7, $647, \circ_{3} 6$ & $6,423,34 \mathrm{I}$ \\
\hline I889... & 8 & 201,787, or 7 & $3,35^{2,708}$ & $364,483,3^{82}$ & I4,760,69I & $5,086,233$ \\
\hline I888... & 7 & I $6 r, 260,335$ & $2,788,000$ & $302,033,066$ & I I, 939,540 & $4,162,745$ \\
\hline I887 ... & 4 & I $58,845,704$ & 2,296, ,OI & 254, IO4,877 & $9,692,425$ & $3,370,346$ \\
\hline I886... & 3 & I $32,674,189$ & $\mathrm{I}, 764,5^{8}$ & I96,694,876 & $7,368,142$ & $2,466,725$ \\
\hline I885 ... & 3 & $93,736,727$ & I,360,376 & I 44, IOI, 632 & $5,530,622$ & I,919,533 \\
\hline $\mathrm{I} 884 \ldots$ & 3 & 89, I 5०,3०2 & $\mathrm{I}, 076,422$ & I08,451,099 & $4,486,6$ I 2 & I,499,432 \\
\hline & 3 & 77, oI 7,326 & $86_{3}, 584$ & $86,321,162$ & $3,276,85^{6}$ & I,029, I60 \\
\hline & 3 & $52,082,28 I$ & 580,364 & 55,5 I 4,768 & $2,233,647$ & 721,072 \\
\hline & 3 & $37,089,5^{22}$ & 359,942 & $32,641,798$ & $I, 608,89 I$ & 541,925 \\
\hline r880... & 3 & $34,768, \circ 35$ & 228,357 & I $9,590,780$ & I, I 55,360 & $430,63 I$ \\
\hline I879... & 3 & $4,956,8 \circ 9$ & 58,185 & $5,334,53$ I & 548,163 & $3^{29}, 575$ \\
\hline & I & I,785,696 & 22,808 & $2,027,888$ & 59,8 I7 & I 1,338 \\
\hline & $\mathbf{I}$ & $967,93^{2}$ & I I, 226 & $x, 0_{3} \circ, 655$ & 28,5 I 7 & 5,296 \\
\hline & I & 727,168 & 4,8 I6 & 443,072 & I 4,495 & $\mathrm{I}, 95^{8}$ \\
\hline Total ... & & $\$ 7,797,291,727$ & & & $\$ 845,385,672$ & $\$ 260,069,267$ \\
\hline
\end{tabular}

This content downloaded from 080.082.077.083 on January 18, 2018 09:32:06 AM All use subject to University of Chicago Press Terms and Conditions (http://www.journals.uchicago.edu/t-and-c). 
For a Payment of 5 Cents per Week With the Burial Benefit Promised Is:

\begin{tabular}{|c|c|c|c|c|c|c|c|c|c|c|c|}
\hline 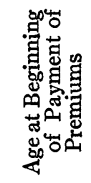 & 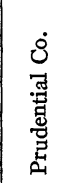 & 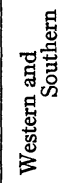 & 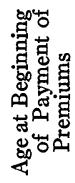 & 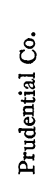 & 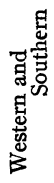 & 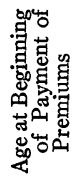 & 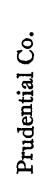 & 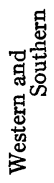 & 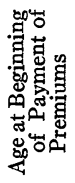 & 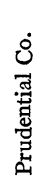 & 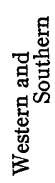 \\
\hline Iо.. & $\$ 120$ & $\$$ I $_{20}$ & 26 & $\$ 74$ & $\$ 75$ & $4 I$ & $\$ 49$ & $\$ 48$ & 56 & $\$ 27$ & $\$ 26$ \\
\hline II. & II 8 & II 5 & 27 & 72 & 72 & 42 & 47 & 46 & 57 & 26 & 25 \\
\hline $12 \ldots$ & II6 & IIO & 28 & $7 \mathrm{I}$ & $7 \mathrm{I}$ & 43 & 45 & 44 & $5^{8}$ & 25 & 24 \\
\hline I3... & II 2 & I06 & 29 & 69 & 69 & 44 & 44 & 42 & 59 & 23 & 22 \\
\hline I $4 .$. & ro8 & IO3 & 30 & 67 & 67 & 45 & 42 & $4 I$ & 60 & 22 & $2 \mathrm{I}$ \\
\hline $15 \%$ & I03 & 100 & $3^{I}$ & 66 & 65 & 46 & $4 \mathrm{I}$ & 40 & 6I & $2 \mathrm{I}$ & 20 \\
\hline I6. & 100 & 98 & $3^{2}$ & 64 & 63 & 47 & 39 & 39 & 62 & 20 & I9 \\
\hline I7. & 94 & 95 & 33 & 62 & $6 \mathrm{I}$ & 48 & $3^{8}$ & 37 & $6_{3}$ & I9 & 18 \\
\hline I8. & 92 & 92 & 34 & 60 & 59 & 49 & 37 & 35 & 64 & I8 & I7 \\
\hline I9. . & 89 & $9 \circ$ & 35 & 59 & 58 & 50 & 35 & 34 & 65 & I7 & I6 \\
\hline 20. & 87 & 88 & $3^{6}$ & 57 & $5^{6}$ & $5^{I}$ & 34 & 33 & 66 & I6 & I5 \\
\hline $2 I \ldots$ & 84 & 85 & 37 & 55 & 54 & $5^{2}$ & $3^{2}$ & $3^{2}$ & 67 & I6 & I4 \\
\hline 22. & 82 & 83 & $3^{8}$ & 54 & 53 & 53 & $3^{I}$ & $3^{I}$ & 68 & I5 & I3 \\
\hline 23. & 80 & $8 \mathrm{I}$ & 39 & $5^{2}$ & $5^{I}$ & 54 & 30 & $3^{\circ}$ & 69 & I4 & I 2 \\
\hline 24. & 78 & 79 & 40 & $5^{\circ}$ & 49 & 55 & 28 & 28 & 70 & I3 & II \\
\hline 25. & 76 & 77 & & & & & & & & & \\
\hline
\end{tabular}

Burial Money at Death of Child, Payable if Policy Has Been in Force the Time Stated ${ }^{x}$

\begin{tabular}{|c|c|c|c|c|c|c|c|c|c|c|c|c|}
\hline Age & $\begin{array}{l}\text { Under } 3 \\
\text { Months }\end{array}$ & $\begin{array}{l}\text { Under } \\
\text { Months }\end{array}$ & $\begin{array}{l}\text { Under } 9 \\
\text { Months }\end{array}$ & $\begin{array}{l}\text { Under } \\
\text { Year }\end{array}$ & $\begin{array}{l}I \\
\text { Year }\end{array}$ & $\stackrel{2}{\text { Years }}$ & Years & Years & Years & $\begin{array}{c}6 \\
\text { Years }\end{array}$ & Years & $\begin{array}{c}8 \\
\text { Years }\end{array}$ \\
\hline & $\$ 8$ & \$10 & $\$ 12$ & $\$ x_{5}$ & $\$ 17$ & $\$ 20$ & $\$ 24$ & $\$ 29$ & $\$ 55$ & $\$ 80$ & $\$ 100$ & $\$ 120$ \\
\hline & 9 & II & $\mathrm{I}_{4}$ & I7 & 20 & 24 & 29 & $5^{I}$ & 75 & 100 & $\mathrm{x} 20$ & $\ldots$ \\
\hline & IO & I3 & I6 & 20 & 24 & 29 & 47 & 70 & 100 & I 20 & $\ldots$ & $\ldots$ \\
\hline 5 & II & $\mathrm{I} 4$ & I8 & 24 & 29 & 43 & 65 & 95 & I 20 & $\ldots$ & $\ldots$ & $\ldots$ \\
\hline & I 2 & I6 & 22 & 29 & 39 & 60 & 90 & I 20 & $\ldots$ & $\cdots$ & $\cdots$ & $\ldots$ \\
\hline$\ldots$ & I4 & I9 & 26 & 35 & 55 & 85 & I 20 & $\cdots$ & $\ldots$ & $\ldots$ & $\ldots$ & $\ldots$ \\
\hline & I6 & 22 & 35 & 50 & 80 & 120 & $\ldots$ & $\ldots$ & $\ldots$ & $\ldots$ & $\ldots$ & $\ldots$ \\
\hline $9 \ldots$ & 20 & 28 & 50 & 75 & I 20 & $\cdots$ & $\ldots$ & $\ldots$ & $\ldots$ & $\ldots$ & $\ldots$ & $\ldots$ \\
\hline
\end{tabular}

× John Hancock, Life Insurance Co. of Virginia, and the Prudential.

Premiuns and Payments of the "Prudential Company of America." Policy of Adults, Entailing a Weekry Premium of:

\begin{tabular}{|c|c|c|c|c|c|c|c|c|c|c|c|c|c|c|c|}
\hline Age & Cts. & $\begin{array}{l}\text { Io } \\
\text { Cts. }\end{array}$ & $\begin{array}{l}\text { I5 } \\
\text { Cts. }\end{array}$ & $\begin{array}{l}20 \\
\text { Cts. }\end{array}$ & $\begin{array}{l}25 \\
\text { Cts. }\end{array}$ & $\begin{array}{l}30 \\
\text { Cts. }\end{array}$ & $\begin{array}{l}35 \\
\text { Cts. }\end{array}$ & $\begin{array}{l}40 \\
\text { Cts. }\end{array}$ & $\begin{array}{l}45 \\
\text { Cts. }\end{array}$ & $\begin{array}{l}50 \\
\text { Cts. }\end{array}$ & $\begin{array}{l}55 \\
\text { Cts. }\end{array}$ & $\begin{array}{l}\text { 6o } \\
\text { Cts. }\end{array}$ & $\begin{array}{l}65 \\
\text { Cts. }\end{array}$ & $\begin{array}{l}\text { 7o } \\
\text { Cts. }\end{array}$ & \\
\hline & \$12O & $\$ 240$ & & $\cdots$ & $\therefore$ & $\cdots$ & $\cdots$ & $\cdots$ & - & 。 & - & o & . & ‥ & \\
\hline I I & II8 & 236 & & $\cdots$ & . . & . & .. & .. & .. & .. & .. & .. & .. & .. & \\
\hline 12 . & I I 6 & $23^{2}$ & $\$ 348$ & & . & . & . & .. & . & , . & . & . & . & . & \\
\hline I3 $\ldots$ & II 2 & 224 & 336 & $\$ 448$ & . & . & .. & .. & . . & .. & .. & . . & .. & . & ; \\
\hline I4. & 108 & 2 I 6 & $3^{24}$ & $43^{2}$ & & . & . & . & . & .. & .. & . & . & $\cdots$ & \$ \\
\hline I5. & IO3 & 206 & 309 & 4I 2 & $\$ 515$ & .. & . & .. & .. & .. & . & .. & .. & . & ส \\
\hline I6. & 100 & 200 & 300 & 400 & 500 & $\cdots$ & . & .. & . & .. & . & .. & . & . & 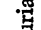 \\
\hline & 94 & I88 & 282 & 376 & 470 & $\$ 564$ & . & .. & . & . & . & .. & . & . & ต \\
\hline & 92 & I84 & 276 & 368 & 460 & $55^{2}$ & & . & . & .. & .. & .. & . & .. & \\
\hline I9 $\ldots$ & 89 & I78 & 267 & $35^{6}$ & 445 & 534 & $\$ 623$ & . & .. & . & .. & . & . & . & \\
\hline
\end{tabular}

This content downloaded from 080.082.077.083 on January 18, 2018 09:32:06 AM All use subject to University of Chicago Press Terms and Conditions (http://www.journals.uchicago.edu/t-and-c). 
Premiums and Payments of the "Prudential Company of America"-Continued

\begin{tabular}{|c|c|c|c|c|c|c|c|c|c|c|c|c|c|c|c|}
\hline Age & Cts. & $\begin{array}{l}\text { Io } \\
\text { Cts. }\end{array}$ & $\begin{array}{l}15 \\
\text { Ctss. }\end{array}$ & Cts. & $\begin{array}{l}25 \\
\text { Cts. }\end{array}$ & Cts. & C.ts. & $\begin{array}{l}40 \\
\text { Cts. }\end{array}$ & $\begin{array}{l}45 \\
\text { Cts. }\end{array}$ & Co & $\begin{array}{l}55 \\
\text { Cts. }\end{array}$ & $\begin{array}{l}60 \\
\text { Cts. }\end{array}$ & $\begin{array}{l}65 \\
\text { Cts. }\end{array}$ & $\begin{array}{l}7 \circ \\
\text { Cts. }\end{array}$ & \\
\hline & $\$ 87$ & $\$ 174$ & $\$ 26 I$ & $\$ 348$ & $\$ 435$ & $\$ 522$ & $\$ 609$ & & & $\cdots$ & $\cdots$ & $\cdots$ & & & \\
\hline $2 I$ & 84 & I68 & 252 & 336 & 420 & 504 & 588 & $\$ 672$ & & .. & .. & $\ldots$ & $\ldots$ & .. & \\
\hline $22 \ldots$ & 82 & I64 & 246 & $3^{28}$ & 4IO & 492 & 574 & 656 & & .. & . & .. & .. & . & \\
\hline $23 \ldots \ldots$ & 80 & I60 & 240 & 320 & 400 & 480 & 560 & 640 & $\$ 720$ & .. & . & .. & .. & .. & \\
\hline $24 \ldots \ldots$ & 78 & I56 & 234 & $3^{\mathrm{I} 2}$ & 390 & 468 & 546 & 624 & 702 & & . & . & . & .. & \\
\hline $25 \ldots \ldots$ & $7^{6}$ & $5^{2}$ & 228 & 304 & 380 & 456 & 532 & 608 & 684 & $\$ 760$ & & .. & . & .. & \\
\hline $26 \ldots$ & 74 & I 48 & 222 & 296 & $37^{\circ}$ & 444 & $5^{18}$ & 592 & 666 & 740 & $\$ 8 \mathrm{I} 4$ & .. & .. & . & \\
\hline $27 \ldots$ & 72 & I44 & 216 & 288 & 360 & $43^{2}$ & $5 \circ 4$ & 576 & 648 & 720 & $79^{2}$ & & .. & .. & \\
\hline 28 . & $7 I$ & I 42 & 2I3 & 284 & 355 & 426 & 497 & 568 & 639 & 710 & $78 \mathrm{I}$ & $\$ 852$ & .. & . & \\
\hline $29 \ldots$ & 69 & I38 & 207 & 276 & 345 & 4I4 & 483 & $55^{2}$ & 621 & 690 & 759 & 828 & .. & .. & \\
\hline $30 \ldots$ & 67 & I34 & 201 & 268 & 335 & 402 & 469 & 536 & 603 & 670 & 537 & 804 & .. & .. & \\
\hline 3 I. & 66 & $\mathrm{I}_{32}$ & I98 & 264 & $33^{\circ}$ & 396 & 462 & $5^{28}$ & 594 & 660 & 726 & 792 & . & .. & \\
\hline $3^{2}$. & 64 & I 28 & I92 & 256 & $3^{20}$ & 384 & 448 & 512 & 576 & 640 & 704 & $7^{68}$ & .. & . & \\
\hline $33 \ldots$ & 62 & I 24 & I86 & 248 & 310 & 372 & 434 & 496 & $55^{8}$ & 620 & 682 & 744 & . & . & \\
\hline $34 \ldots$ & 60 & 120 & 180 & 240 & 300 & $3^{60}$ & $3^{20}$ & 480 & 540 & 600 & 660 & 720 & .. & .. & \\
\hline $35 \ldots$ & 59 & II8 & I77 & 236 & 295 & 354 & 4I 3 & 472 & $53 \mathrm{I}$ & 590 & 649 & 708 & . & .. & \\
\hline $3^{6} \ldots$ & 57 & II 4 & I $7 \mathrm{I}$ & 228 & 285 & 342 & 399 & $45^{6}$ & $5^{\mathrm{I}} 3$ & 570 & 627 & 684 & .. & . & \\
\hline $37 \ldots \ldots$ & 55 & I IO & 165 & 220 & 275 & 230 & 385 & 440 & 495 & 550 & 605 & 660 & . & . & \\
\hline $3^{8} \ldots \ldots$ & 54 & IO8 & I62 & 216 & 270 & 324 & 378 & 432 & 486 & 540 & 594 & 648 & .. & . & \\
\hline $39 \ldots$ & $5^{2}$ & IO4 & I56 & 208 & 260 & $3^{12}$ & $3^{64}$ & $4 \mathrm{I} 6$ & 468 & $5^{20}$ & 572 & 624 & .. & . & \\
\hline $40 \ldots$ & 50 & IOO & I50 & 200 & $25^{\circ}$ & 300 & 350 & 400 & $45^{\circ}$ & 500 & $55^{\circ}$ & 600 & .. & .. & \\
\hline 4I. & 49 & 98 & I47 & I96 & 245 & 294 & 343 & 392 & 44I & 490 & 539 & 588 & & .. & \\
\hline $42 \ldots$ & 47 & 94 & I4I & I88 & 235 & 282 & $3^{29}$ & 376 & 423 & 470 & 5 I7 & $5^{64}$ & $\$ 6 \mathrm{II}$ & . & \\
\hline $43 \ldots \ldots$ & 45 & 90 & I35 & I80 & 225 & 270 & 315 & 360 & 405 & 450 & 495 & 54 잉 & $5^{8} 5$ & . & 节 \\
\hline 44. & 44 & 48 & I32 & 工 76 & 220 & 264 & 308 & $35^{2}$ & 396 & 440 & 484 & $5^{28}$ & 572 & & \\
\hline $45 \ldots \ldots$ & 42 & 84 & I 26 & I68 & 210 & 252 & 294 & $33^{6}$ & 378 & 420 & 462 & 504 & 546 & $\$ 588$ & ต \\
\hline 46. & $4 \mathrm{I}$ & 82 & I 23 & I64 & 205 & 246 & 287 & $3^{28}$ & $3^{69}$ & 4IO & $45^{\mathrm{I}}$ & 492 & 533 & 574 & F \\
\hline $47 \ldots$ & 39 & 78 & II 7 & I56 & I95 & 234 & 273 & $3^{\mathrm{I} 2}$ & $35^{\mathrm{I}}$ & 390 & 429 & 468 & $5 \circ 7$ & 546 & 5 \\
\hline & $3^{8}$ & 76 & II4 & I 52 & I90 & 228 & 266 & 304 & 342 & $3^{80}$ & 4I8 & $45^{6}$ & 494 & $53^{2}$ & \\
\hline $49 \ldots \ldots$ & 37 & 74 & III & I48 & 185 & 222 & 259 & 296 & 333 & 370 & 407 & 444 & $48 \mathrm{I}$ & $5^{10}$ & \\
\hline & 35 & 70 & I05 & I40 & I75 & 210 & 245 & 280 & 315 & 350 & 385 & 420 & 455 & 490 & \\
\hline $51 \ldots$ & 34 & 68 & I02 & I36 & I 70 & 204 & 238 & 272 & 306 & 340 & 374 & 408 & 442 & $\cdots$ & \\
\hline & $3^{2}$ & 64 & 96 & I 28 & I60 & I92 & 224 & 256 & 288 & 320 & 352 & 384 & 4I6 & . & \\
\hline 53 & $3^{I}$ & 62 & 93 & I 24 & I55 & I86 & 217 & 248 & 279 & $3^{\text {Io }}$ & $34 \mathrm{I}$ & 372 & 403 & .. & \\
\hline 54 & 30 & 60 & 90 & I 20 & 150 & I80 & 210 & 240 & 270 & 300 & 330 & $3^{60}$ & 390 & .. & \\
\hline & 28 & $5^{6}$ & 84 & I I 2 & I40 & I68 & I96 & 224 & 252 & 280 & 308 & $33^{6}$ & $3^{64}$ & . & \\
\hline $5^{6}$ & 27 & 54 & $8 \mathrm{I}$ & 108 & I35 & I62 & I89 & 216 & 243 & 270 & 297 & $\ldots$ & $\cdots$ & .. & \\
\hline 57 & 26 & $5^{2}$ & 78 & I04 & I30 & I56 & I82 & 208 & 234 & 260 & 286 & .. & . & . & \\
\hline & 25 & 50 & 75 & IOO & I25 & I 50 & I75 & 200 & 225 & 250 & 275 & . & . & .. & \\
\hline 59 & 23 & 46 & 69 & 92 & II 5 & I 38 & I6I & I84 & 207 & 230 & 253 & . & .. & . . & \\
\hline & 22 & 44 & 66 & 88 & IIO & I32 & I54 & I76 & I98 & 220 & 242 & . & $\cdots$ & - & \\
\hline 6 I $\ldots$. & $2 \mathrm{I}$ & 42 & 63 & 84 & I05 & I 26 & I47 & I68 & I89 & . & .. & .. & . & & \\
\hline 62. & 20 & 4잉 & 60 & 80 & 100 & I 20 & I40 & I60 & I80 & . & . & .. & . & . & \\
\hline 63 & I9 & $3^{8}$ & 57 & 76 & 95 & II 4 & I33 & $\mathrm{I}^{2}$ & $\mathrm{I}_{7} \mathrm{I}$ & . & .. & .. & . & $\cdots$ & \\
\hline 64 & I8 & 36 & 54 & 72 & 90 & I08 & I 26 & I44 & I62 & & & & & & \\
\hline & I7 & 34 & 5I & 68 & 85 & IO2 & I I9 & \multirow{6}{*}{\multicolumn{7}{|c|}{ See footnote I }} & \\
\hline & I6 & $3^{2}$ & 48 & 64 & 80 & 96 & II 2 & & & & & & & & \\
\hline & I6 & $3^{2}$ & 48 & 64 & 80 & 96 & II 2 & & & & & & & & \\
\hline & I5 & 3 이 & 45 & 60 & 75 & 90 & IO5 & & & & & & & & \\
\hline & I4 & 28 & 42 & $5^{6}$ & 70 & 84 & 98 & & & & & & & & \\
\hline $70 \ldots$ & I3 & 26 & 39 & $5^{2}$ & 65 & 78 & 9I & & & & & & & & \\
\hline
\end{tabular}

$x$ One-fourth of the death benefit in the first six months; one-half of death benefit in the second six months; full benefit after one year of insurance. 
Infantile Table Entailing a Weekly Premium of 5 Cents

\begin{tabular}{|c|c|c|c|c|c|c|c|c|}
\hline \multirow{2}{*}{ Payments After Following Period } & \multicolumn{8}{|c|}{ Age at Taking Out Policy } \\
\hline & 2 & 3 & 4 & 5 & 6 & 7 & 8 & 9 \\
\hline 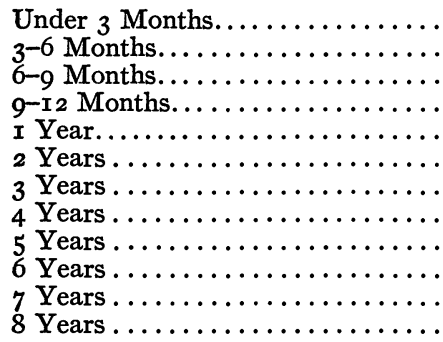 & $\begin{array}{r}\$ 8 \\
10 \\
12 \\
15 \\
17 \\
20 \\
24 \\
29 \\
55 \\
80 \\
100 \\
120\end{array}$ & $\begin{array}{r}\$ 9 \\
\text { II } \\
\text { I4 } \\
\text { I7 } \\
20 \\
24 \\
29 \\
51 \\
75 \\
100 \\
120 \\
\ldots\end{array}$ & $\begin{array}{r}\$ 10 \\
\text { 13 } \\
\text { I6 } \\
20 \\
24 \\
29 \\
47 \\
70 \\
\text { 100 } \\
\text { I20 } \\
\cdots \\
\cdots\end{array}$ & $\begin{array}{r}\$ \text { II } \\
\text { I4 } \\
\text { I8 } \\
24 \\
29 \\
43 \\
65 \\
95 \\
\text { I20 } \\
\cdots \\
\cdots \\
\cdots\end{array}$ & \begin{tabular}{|r} 
I2 \\
16 \\
22 \\
29 \\
39 \\
60 \\
90 \\
I20 \\
$\cdots$ \\
$\cdots$ \\
$\cdots$ \\
$\cdots$
\end{tabular} & $\begin{array}{r}\$ 14 \\
19 \\
26 \\
35 \\
55 \\
85 \\
120 \\
\cdots \\
\cdots \\
\cdots \\
\cdots \\
\cdots\end{array}$ & $\begin{array}{r}\$ 16 \\
22 \\
35 \\
50 \\
80 \\
120 \\
\ldots \\
\cdots \\
\cdots \\
\cdots \\
\ldots \\
\ldots\end{array}$ & $\mid \begin{array}{r}\$ 20 \\
28 \\
50 \\
75 \\
\mathrm{120} \\
\cdots \\
\cdots \\
\cdots \\
\cdots \\
\cdots \\
\cdots \\
\cdots\end{array}$ \\
\hline
\end{tabular}

A weekly premium over ro cents is not collected.

Premium for Adults on a Policy Carrying a $\$ 500$ Death Benefit 2

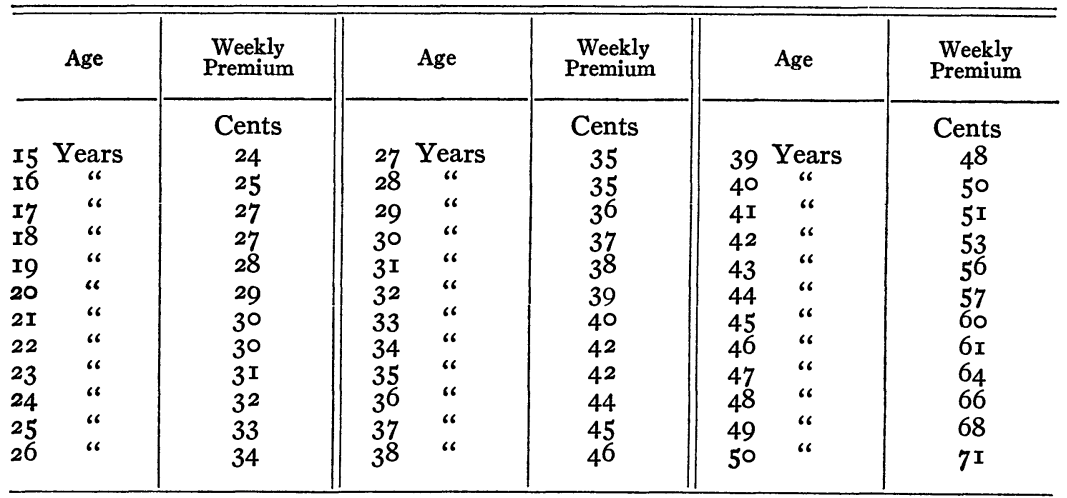

2 During the first six months $\$ 125 ; \$ 250$ during the second six months, and full benefit $(\$ 500)$ after one year of insurance.

Value of industrial insurance.-We may freely admit that the claim of the companies that they offer real benefits to lowpaid workmen has considerable foundation in fact, and they are entitled to consideration. It is incredible that such a vast business should rest upon unmitigated falsehood and injustice. The companies are right in their claim that no considerable number of workmen of this level will voluntarily insure, even if rates are low, and that solicitation by agents is costly and must be charged in the premiums. They are right in claiming that the benefits have often spared the poor family the shame of a pauper 
funeral; that family feeling and affection are fostered; that a spirit of independence and self-respect is maintained; and these are not insignificant advantages, although we may think they cost too dear and may be better gained in other ways.

Pauper burials, although not an accurate measure of the distress of the period, reached an average rate of 20 per I0,000 of population. During I88I to 1885 the rate for 18 cities was 18.5 against an average of 12.9 during the five years 1897 to Igor. $^{2}$

Hoffman (History of the Prudential Insurance Co., p. 308) presents a study of pauper burials in ten cities (New York, Boston, Newark, Cincinnati, Baltimore, Indianapolis, Minneapolis, Cambridge, Worcester, and Charlotte), and finds that the rate of pauper burials in I00,000 population was, in 1880 to I884, 2 IO, and fell in I895 to I899 to I56, after the industrial companies had had time to establish the custom of burial insurance on a general scale. It is impossible to discover all the causes of this decrease, but we may admit a large influence from burial insurance. They have unintentionally rendered another service to the cause of a rational system of insurance which the future will develop; they have shown that voluntary systems are costly and inadequate and that in all probability obligatory measures alone will bring such benefits within the power of low-paid workmen. But while we may try to be just to the companies, and may admit that they have responded to a universal demand of wage-earners, we have still to inquire whether the good has not been purchased at too great a price, and whether a more economical system is not possible. The premiums are relatively high and the benefits extremely low. The poorer wage-earners must content themselves with extremely small returns for their enormous sacrifices. Numerous workers in charitable societies complain that after the burial fees are paid there is nothing left for savings; that while thrift may be cultivated in one direction the benefits paid at death in a lump sum lead almost universally and inevitably to extravagant funerals and display, so that the insurance company, their agents, and the undertakers profit by

${ }^{2}$ J. F. Dryden, $A$ Quarter Century of Industrial Insurance in the United States, p. 8. 
the losses of those who can least afford such expenditures. President Hegemann has stated that in 86 per cent. of cases investigated the expenses of sickness and burial exceeded the benefits paid, and that the average sum paid on infantile policies in the year 1897 was only $\$ 25.83$.

A very material consideration in this connection is that while the burial-benefit companies absorb the greater part of the available resources for insurance purposes in families of small income, they by no means cover all the insurance needs of such families and, perhaps, not the most pressing. The attempt was made in the earlier history of these companies to provide sickness insurance, but the effort failed and had to be abandoned. President Dryden, in his account of this movement, says that a company which has its business scattered over a wide territory, and must act through salaried agents, cannot undertake sickness insurance, and that this form of insurance is possible only in brotherhoods or small groups where the members know each other and can detect and discipline malingerers. ${ }^{3}$ In the same way it can be shown that this form of organization cannot conduct accident insurance, without radical changes of method; and, indeed, it would be grossly unjust, as we have elsewhere shown, to lay this burden on the poorly paid employees. We must conclude, therefore, that these companies are restricted to a very limited field of industrial insurance, that they render a necessary service at enormous and burdensome cost, and that this cost is so heavy as to hinder both savings and insurance of a desirable kind.

A recent and valuable study of the operation of certain companies not named has been published in Bulletin 67 of the Bureau of Labor, November, 1906, by Mr. S. E. Forman. In this intensive study of a particular city we see the working and effect of the system at large, although here some of the worst features appear in aggravated form. Washington, as the capital city, has few manufactures and relatively a large number of personal servants and persons employed in ministering to personal convenience of visitors and residents. The ratio of poorly paid negroes living on fluctuating income, with high rate of sickness

'Inception and Early Problems of Industrial Insurance, pp. I6, 23. 
and mortality, is very high, and their housing conditions are generally bad. Among these the industrial insurance companies which raise funds by levying assessments are popular. The assessment companies are not akin to the fraternal societies elsewhere discussed, but are companies for profit of the directors and stockholders. They are distinguished also from the industrial-insurance companies considered already in the fact that they carry on sickness and accident insurance with burial benefits added. They are not legally required to carry a reserve fund; they collect the premiums by the costly method of weekly visits, or sometimes by monthly visits; and the contract permits them to levy assessments to meet deficits, although in fact competition with other companies prevents them from exercising this right under ordinary circumstances; if the weekly premium is five cents then the yearly premium will be about $\$ 2.60$. The policy promises sickness, accident, and death benefits, although the forms of contracts are varied. Twelve of these companies, on December 31, I903, had in force 28,92 I policies of this type. Forman has shown that those insured in these companies must pay very dearly for their insurance-at least 75 per cent. more than those insured in the regular companies, and also more than those insured in the ordinary industrial insurance companies which offer accident and sickness benefits. If we compare the insured in the assessment companies with those insured in the regular companies we find that they must lose, that is in excessive premiums pay more. We may cite the conclusions:

The price of regular industrial insurance in all its forms has been seen to be very much higher than that of ordinary insurance. An analysis of the insurance business of the District of Columbia for 1903 furnishes some measure of the losses to the policy-holders resulting from the purchase of life insurance on the weekly payment or industrial plan when compared with the cost of ordinary insurance. The rate of premiums charged differs according to the form of the policy, but an examination of the several tables which have been given would seem to justify the statement that on the average the charge for regular industrial insurance is at least 75 per cent. higher than that for ordinary insurance. If the amounts collected for premiums from the regular industrial policy-holders $(\$ 864,059.6 \mathrm{r})$ could have been paid in annual payments and could have purchased insurance at the rates charged by the ordinary companies, $\$ 40,250,227$ would have been secured by 
the industrial policy-holders instead of $\$ 23,000,130$, the amount actually secured under the industrial plan. This represents an apparent loss in insurance protection to the industrial policy-holders of $\$ 17,250,000$, or, if it be measured in premium payments, an apparent loss of over $\$ 370,000$ upon premium payments of the year.

If now we turn to the combination schemes offered by the assessment companies we find that similar policies of ordinary companies furnish 300 per cent. more insurance for the same money. Placing the ascertained facts together for the poorest people of Washington, we may accept this estimate of loss:

Losses measured by amount of insurance carried-

a) Regular industrial insurance $\ldots \ldots \ldots \ldots \ldots \ldots 17,250,000$

b) Assessment industrial insurance $\ldots \ldots \ldots \ldots, 3,375,000$

Total $\$ 20,625,000$

Or, if the losses be measured in premium payments made during the year in excess of what would have been required to purchase the same amount of insurance if the premiums could have been paid in yearly payments in ordinary companies, they may be expressed as follows:

Losses measured by excessive premiums-

a) Regular industrial insurance $\ldots \ldots \ldots \ldots \ldots \ldots . \$ 370,000$

b) Assessment industrial insurance $\ldots \ldots \ldots \ldots \ldots+120,000$

Total .............................. $\$ 490,000$

Of course the facts set forth above tell nothing about the reasonableness or unreasonableness of the cost of either ordinary insurance or of industrial insurance. They simply show what the cost of industrial insurance is and how much greater that cost is than the cost of ordinary insurance, and illustrate, as did the study of conditions of living among the poor, that the smaller the earning power of a wage-earner, the smaller also is the purchasing power of each of his dollars.

The facts already recited have long been familiar to students and to visitors among the poor of our cities, and many schemes for mitigating the evils have been debated, thus far without result. The older counsel was to encourage saving deposits and to assist the people to utilize very small savings for this end. But to this plan there are very grave objections, since it is an attempt to lay the burden of industrial risk altogether on the poor and compel them to carry the accident insurance burden which all admit should be borne by the business which causes the risk. 
Furthermore the sum which can by any possibility be saved by unskilled workpeople is utterly inadequate at any time and especially during the first years of married life when the expense of rearing children increases and consumes all earnings.

The chief causes of the extravagant insurance premiums to the poor are: (a) the unfair part of the receipts from premiums and interest which is kept by the chief officers of the companies from the dividends of the insured; $(b)$ the excessively high salaries of the officers of administration; $(c)$ above all the fees to agents for soliciting insurance under the weekly collection plan. The first and second causes of waste may be to some extent reduced by the legislative and administrative action of the states, by publicity of accounts, and by inspections and rules of management. But not in any such direct way can political means reduce the third and most important cause of waste of the contributions of low-paid workingmen. If we are ever to place the business of industrial insurance on a fair and economic basis the agent must be dismissed; all other means of relief are relatively insignificant. Assuming that every director is honorable, that expenses of administration are reduced to a minimum, that the agents themselves are paid a mere pittance, yet the system itself must necessarily absorb a very great sum from the hard-won earnings of the working-people. This argument has been urged by the advocates of the savings-bank method of industrial insurance in Massachusetts. A society ${ }^{4}$ has been formed in Boston for the purpose of securing legislation permitting the savings banks to go into the life-insurance business; and the necessary law has been passed. Among the founders of this association are numbered men of education, philanthropy, and business standing; some of them would be glad to help introduce compulsory insurance in some form, but are discouraged from making efforts in that direction by the apathy of the public, the failure of the bill offered in 1904, and by the constitutional and economic obstacles which confront all such attempts in this country. Some of the savings banks have declared their readiness, in case they are empowered by law, to try the proposed experiment. Under the

«The Savings Bank Insurance League. 
plan proposed there would be no expense of solicitation by agents; the commodity would be offered, and then the banks would depend on the education of the people to induce them to take advantage of the opportunity of insuring themselves at bare cost. It is argued by the friends of this movement that the savings banks not only in Massachusetts but elsewhere have won the confidence of the country by their honest and careful management of the deposits, and in some instances the administrators, with the exception of a few salaried officers, perform their duties without charge and for the public good. Thus the savings banks, especially in Massachusetts, seem to be the most promising agencies for cheap insurance.

On the other hand the representatives and managers of the regular, orthodox life-insurance companies think they have discovered the Achilles-heel of this scheme. They assert that comparatively few persons, least of all the very poor who most need relief, can be induced to apply voluntarily for insurance without the persistent labor of agents. The employment and payment of agents is a necessary and legitimate expense, since without it working-people must go without the needed benefits. It is asserted by these advocates of present methods that the founders of the new association, however estimable and amiable, must lack knowledge of the business and the history of life insurance, that they are mere theorists and impractical. In support of this contention they cite the experience of the British companies who have tried a similar scheme, the old Equitable, the London Life, and the Metropolitan of London. Still more striking is the example of the industrial insurance societies whose purpose is to guarantee burial money for working-people. The British Postoffice Department has offered small policies for forty years at low cost. These policies are written at local post-offices and the premiums may be paid in weekly instalments. At the end of the year 1904, after forty years' trial, the government insurance office had in force only 12,875 policies of this kind; while the Pearl Life Assurance Company of London, which began operations only a year earlier than the post-office, had in force 2,320,463 policies, and the Refuge Assurance Company of the 
same age as the Pearl, had 2,628,650 industrial policies in force. The Prudential Assurance Company of London, only a little older than the post-office department of insurance, had in force at the end of the year 1904 between fifteen and sixteen million policies. During the year I904 the post-office, with its 23,068 branch offices wrote only $5^{\mathrm{I}} 7$ new policies. The Prudential of London in the same year wrote $7 \mathrm{I}, 700$ industrial policies. The conclusion of these experts and representatives of the insurance companies therefore virtually is that there is no relief for the working-people; the only outlook is that they must continue to bear this heavy burden.

Of course the philanthropists of Massachusetts may be able to set in motion educational agencies to reverse this condition and win customers to their savings-bank insurance companies. A1ready the powerful associated charities are considering methods of co-operation with the insurance associations; and with their fine organization of friendly visitors they might accomplish much; how much, only trial can reveal. . But in any case these associations must still leave the great problems of accident and sickness insurance at one side; they cannot solve these problems, for only compulsory insurance can ever, at one stroke, make insurance even general.

\section{CASUALTY INSURANCE COMPANIES}

In the absence of social organization by the states, certain accident insurance companies have entered into competition with trade unions and mutual benefit societies to furnish the desired accident and sickness insurance for wage-earners. We have not yet at hand satisfactory statistics of the operations of these companies, and we are told by some of them that they dare not let rival companies even see their reports on account of the exigencies of competition. The reports which have been published do not always distinguish the economic classes of their clients, and so do not inform us what number of wage-earners are included. Skilled artisans and well-paid mechanics may be able to pay for a fair amount of accident and sickness insurance, but the rates are prohibitive for those on bare living-wages, and these are in 
the great majority. Even when insurance is taken there is common and growing complaint that the contracts are narrow and narrowly interpreted in settlements. Thus it is claimed that many diseases are included which rarely occur and many omitted which are very common; so that in reality more is promised than is paid. It is asserted that there are so many technical clauses modifying the agreements that no man can know in advance what his claim actually is. But the need of insurance is so widely and keenly felt, and the misery of being without protection is so intolerable, that the business of these companies is growing and is already considerable. Not seldom the employers are disposed to assist the introduction of this form of insurance in their establishments, since they know its value to the men and realize that men who are insured are somewhat less inclined to sue for damages in case of injury if they have some benefits coming in during disability. The companies began with accident insurance, but the demand for sickness insurance led some companies to offer this and competition is driving other companies to follow their example.

The workmen's collective policy.-The essential feature of this plan is to include all the employees of a firm or corporation in a single contract which insures them against loss by reason of accident or accident and sickness. The employer pays a premium which is based on the number of employees, the hazard of the occupation, and the amount of wages. The insurance company agrees to pay indemnities according to a graduated scale. Then the employer makes a contract with his employees according to which he is authorized by them to retain a weekly sum from their wages to reimburse him for payment of premiums. Rarely, the employer pays a part or even all the premiums himself without taking anything from wages. The premium advanced is based provisionally on the estimated number of workmen and the amount of wages for the coming year; if at the end of the year it appears that the force has been increased a supplementary sum must be paid the company insuring; and if the payroll shows that the premium advanced was too large the insuring company returns the excess. The employer acts as trustee of the men and 
is paid for his trouble usually 5 per cent. for cost of collecting premiums. If, as sometimes happens, the employer insures himself against damage suits, another 5 per cent. is deducted from the premium. Both forms of insurance may be covered in one policy. This form has suggested some of the features of the bill proposed by the Illinois Industrial Insurance Commission.

A few examples are given of various forms of insurance of large numbers of employees. The General Accident Insurance Company of Philadelphia deposits $\$ 100,000$ with the Pennsylvania Insurance Department to give a guarantee of all contracts. In its industrial department it writes policies for workingmen's indemnity, which it describes as a collective policy issued to the employer as trustee for his employees, furnishing health and accident insurance for monthly premiums, paying monthly benefits to employees for loss of time caused by accident, not to exceed fiftytwo consecutive weeks, no matter when or how the accident happens, whether in factory, going to or from work, or on recreation. Substantial benefit is paid if the insured is killed by accident, or for the loss of one or more limbs or eyes. Sick benefits are paid for every disease to which flesh is heir, while the insured is confined to the house, after the policy has been in force thirty days for a limit of six months, with the exception of rheumatism, paralysis, tuberculosis, Bright's disease, for which full indemnity is paid for a limit of two months in any one year. In addition, full medical or surgical attention is given, whether disabled or not. Inducements are offered to the employers to encourage the introduction of their plan in shops and mills. The language of the advertisement is quoted:

This form of insurance is 50 per cent. cheaper than any other form of workmen's collective insurance that has ever been offered. We save from 25 to 50 per cent. on the cost of your employers' liability insurance by introducing this form of insurance in your plant.

In the policy occurs a clause which shows how cost of employers' liability is reduced:

The acceptance by an employee, or any other person who may be entitled thereto, of a benefit under this policy for injury or death of the employee, shall operate as a release of all claims for damages against the assured arising from such injury or death which could be made by or through the 
employee, or any other person, and the person so accepting the benefit shall execute such further instruments as may be necessary formally to evidence such acquittance.

This form of policy is increasingly objectionable to workingmen, particularly where the employer contributes little or nothing to the premium, and it is becoming every day more unpopular. The workmen declare that it is unjust to ask them to make heavy sacrifices in loss of a portion of wages to build up a fund for insurance and then deprive them of enjoying it in case of injury unless they sign away their common-law rights to sue the employer for damages due to his negligence. This objection would lose its point if the employer contributed a sum substantially equal to that he must pay to protect himeslf from loss under the liability law.

The New Amsterdam Casualty Co. has had some experience with industrial insurance of the kind under consideration. The agreement with the insured is to indemnify against loss from bodily injuries sustained by an employee or employees of the insuring employer through external, violent, and accidental means, while actually engaged in the occupations and at the places mentioned in the schedule, and resulting from the operation of the trade or business described in the schedule. The president of this company says of this form of policy:

Workmen's collective insurance is wholesale accident insurance, the policy running to the employer, and the protection thereunder being for the workmen whether the employer be legally liable for the injuries or not. In some cases the employer assesses the premium back on the men by deducting all or part of it from the wages, a certain percentage being deducted on each pay day. The insurance company, however, assumes the full burden of reimbursing the men for injuries such as are covered by the policy. ${ }^{5}$

The net premiums of this company by years have been as follows :

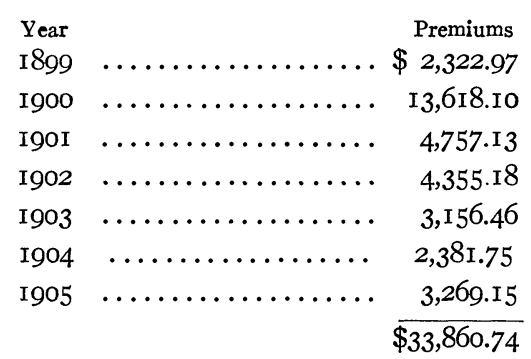

${ }^{5}$ Letter of Mr. W. F. Moore, April 27, 1906. 
Insurance of individual workmen.-Some of the casualty companies do not attempt to do business among working-men but confine themselves to selected risks with persons of larger income who pay yearly and thus receive the benefit of lower cost for administration and for being in a less hazardous class. Companies which insure working-men must, as a rule, collect the premiums monthly in small amounts. This increases cost of solicitation and collection which must be charged in the premiums. And since the policy must be renewed each year the cost of solicitation is still more increased. Under the plan of insuring individual workmen the company deals with the insured more or less directly, although arrangements are sometimes made with employers to collect the premiums, in which case the collective form is closely approached. Sickness insurance is usually connected with accident policies and cannot otherwise be obtained.

The Standard Life and Accident Insurance Company, of Detroit, Mich., may be used for illustration. (Vide Instructions of March, I906.) This company employs agents to solicit business, and it has local agents in towns and cities, not for the purpose of calling upon the policy-holders but for furnishing convenient means of collecting premiums. A drug store is preferred for a local agency because it is open in the evening. A commission of 5 per cent. is allowed the collector for receipting and remitting the premitums of policy-holders. Women between the ages of eighteen and forty-five engaged in occupations from which they derive a regular income, and on which they depend for support, will be granted insurance in the sum of $\$ 25$ per month accident and illness indemnity with $\$ 200$ accidental death insurance for a premium of $\$ \mathrm{I}$ per month. Those desiring larger indemnities must be classified according to occupation and pay a premium 50 per cent. higher than that specified in the rate table. In no event will they be written for more than $\$ 50$ per month accident and $\$ 40$ per month illness benefit, nor to exceed threefourths of their average income. Over-insurance is avoided in all cases. The indemnity should not exceed three-fourths of the average actual money value of the insured's time, or of the amount of his monthly salary or wages. The insurance is not 
forfeited by change of occupation, but in the event of receiving an injury when engaged in a more hazardous occupation, the sum insured and the monthly indemnity will be for such amounts as the premium paid shall be sufficient to purchase at the rates fixed by the company tables for such increased hazard of occupation. Insurance is not written on any person who is under seventeen years or over sixty. Applicants between fifty and sixty pay 50 per cent. additional premium. There is no graduation of premiums between seventeen and fifty years. The beneficiary must have an insurable interest in the life of the insured, as wife, child, parent, or other heir-at-law, or must be a dependent relative, fiancée, or a charitable institution. In case of accidental injury to, or sickness of, any person insured in this company, for which a claim is likely to be made, immediate notice must be given. Payments of claims are made by check to the insured or agent after investigation and adjustment. Surgeons are appointed in localities where the business is large enough to warrant such an appointment, and their duty is to protect the interests of the company. Premiums are payable monthly in advance at the home or branch office. The detailed definition of accident indemnity is significant. Full accident indemnity is paid for accidental death, loss of one or more limbs, or both eyes, and for loss of time, resulting from bodily injuries caused solely by external, violent, and accidental means such as dislocation, fractures, broken bones, bruises, cuts, shot wounds, crushing or mangling, burns or scalds, bites of dogs and serpents, stroke of lightning, drowning, or injuries produced by falls, or any other purely accidental injury happening to the insured in any of the lawful vocations of life, whether such accident happen at home, or in the office, going to or from work, in the store, factory, shop, mill, yard, or on the street or farm, traveling on passenger trains, street cars, steamboats, walking, riding, driving, boating, etc., but will not be paid except at one-fifth the indemnity otherwise stated, in case of disappearance, or suicide, sane or insane; nor for any injury, fatal or non-fatal, resulting wholly or partly, directly or indirectly, from intoxication or the use of narcotics from, or while violating law, war risks, inhalation of gas, vapor, or 
anaesthetic, voluntary over-exertion, wilful or gross negligence, unnecessary exposure to apparent danger, surgical operations not necessitated solely by injury and made within. ninety days after the accident. Sick indemnity is paid for the time, after the first week, that the insured is necessarily confined to the house by reason of any disease or illness, except rheumatism, paralysis, lumbago or lame back, hernia, orchitis, sciatica, insanity, dementia, and venereal diseases, which would be covered by onefifth the regular indemnity. Some risks are prohibited; the following will not be accepted for insurance on any terms: Persons who are blind, deaf, dumb, feeble-minded, cripples, intemperate, disreputable, or persons without visible means of support, those engaged in gambling, in handling highly inflammable or highly explosive material in factory or warehouse, aeronauting, driving, submarine working, rubber grinding or mixing; electricians handling live wires or working about machines where it is possible to receive a direct current of 500 volts, or an alternating current of 250 volts; professional baseball players, laborers or machinists employed in constructing tunnels or caissons; soldiers or sailors engaged in active warfare; blasters, insane persons, persons compelled to use a crutch, subject to fits or vertigo, who have suffered from paralysis, or are paralyzed, or have any deformity that will in any way hinder the regular duties of life; powder-makers, circus performers, fishermen on the sea, fireworks' employees and employers, cartridge makers, football players.

There are ten classes of risks; select, preferred, extrapreferred, ordinary, extra-ordinary, medium, extra-medium, hazardous, special hazardous, and there is a table of indemnity and cost for each class. Thus the table of indemnity and cost for the select class is :

\begin{tabular}{|c|c|c|c|c|c|c|}
\hline & $\begin{array}{l}\text { Monthly } \\
\text { Accident } \\
\text { Indemnity }\end{array}$ & $\begin{array}{l}\text { Monthly } \\
\text { Illness } \\
\text { Indemnity }\end{array}$ & $\begin{array}{l}\text { Accidental } \\
\text { Death or Loss } \\
\text { of Two Limbs } \\
\text { or Both Eyes }\end{array}$ & $\begin{array}{c}\text { Loss of } \\
\text { One Limb }\end{array}$ & $\begin{array}{l}\text { Cost per } \\
\text { Month, Regu- } \\
\text { lar Policy }\end{array}$ & $\begin{array}{l}\text { Cost per } \\
\text { Month, Special } \\
\text { Policy }\end{array}$ \\
\hline $\begin{array}{l}1 \ldots \ldots \\
2 \ldots \ldots \\
3 \ldots \ldots \\
4 \ldots \ldots \\
5 \ldots \ldots \\
6 \ldots \ldots \\
7 \ldots \ldots\end{array}$ & $\begin{array}{r}\$ 50 \\
60 \\
60 \\
70 \\
80 \\
80 \\
100\end{array}$ & $\begin{array}{r}\$ 50 \\
40 \\
60 \\
60 \\
50 \\
60 \\
60\end{array}$ & $\begin{array}{r}\$ 500 \\
600 \\
600 \\
700 \\
800 \\
800 \\
1000\end{array}$ & $\begin{array}{r}\$ 250 \\
300 \\
300 \\
350 \\
400 \\
400 \\
500\end{array}$ & $\begin{array}{r}\$ \mathrm{r} .00 \\
\mathrm{r} .00 \\
\mathrm{r} .25 \\
\mathrm{r} .40 \\
\mathrm{r} .5 \circ \\
\mathrm{r} .60 \\
\mathrm{r} .75\end{array}$ & $\begin{array}{r}\$ \mathrm{I} .50 \\
\mathrm{I} .50 \\
\mathrm{I} .75 \\
\mathrm{I} .90 \\
2.00 \\
2.10 \\
2.25\end{array}$ \\
\hline
\end{tabular}


Additional accidental death insurance will be written in this class at 25 cents per month for each $\$ 500$. Applicants over fifty years of age must pay 50 per cent. more.

The table of the special hazardous class is :

\begin{tabular}{|c|c|c|c|c|c|}
\hline $\begin{array}{l}\text { Monthly } \\
\text { Accident } \\
\text { Indemnity }\end{array}$ & $\begin{array}{l}\text { Monthly } \\
\text { Illness } \\
\text { Indemnity }\end{array}$ & $\begin{array}{c}\text { Accidental } \\
\text { Death or Loss } \\
\text { of Two Limbs } \\
\text { or Both Eyes }\end{array}$ & $\begin{array}{l}\text { Loss of One } \\
\text { Limb }\end{array}$ & $\begin{array}{l}\text { Cost per } \\
\text { Month, Regu- } \\
\text { lar Policy }\end{array}$ & $\begin{array}{l}\text { Cost per } \\
\text { Month, Special } \\
\text { Policy }\end{array}$ \\
\hline$\$ \mathrm{I}_{5}$ & $\$ \mathrm{I}_{5}$ & $\$$ Iroo & $\$ 50$ & $\$ 1.00$ & $\$ 1.25$ \\
\hline 20 & 20 & IOO & 50 & I.40 & I. 65 \\
\hline 25 & 25 & 100 & 50 & I.75 & 2.00 \\
\hline 30 & 30 & IOO & 50 & 2.25 & 2.50 \\
\hline 35 & 35 & roo & 50 & 2.60 & 3.10 \\
\hline 40 & 40 & roo & 50 & 3.00 & 3.50 \\
\hline
\end{tabular}

Additional accidental death insurance will be written in this class at $\$ 1.00$ per month for each $\$ 500$, with a limit of $\$ 1,000$. Male applicants over fifty years of age must add 50 per cent. to the above premiums.

This company has a special arrangement for "railroad instalment insurance." No person may be insured under the accident policy under eighteen or over sixty-five years of age, nor in the sickness policy over sixty years of age. The rates are the same for all ages. The rates for locomotive engineers are:

For $\$ 1,000$ death benefit and $\$ 5$ weekly indemnity, annual premium, $\$ 1800$ For $\$ 1,000$ death benefit and $\$ 20$ weekly indemnity, annual premium, $\$ 50.40$ For $\$ 2,000$ death benefit and $\$ 20$ weekly indemnity, annual premium, $\$ 36.00$ For $\$ 2,000$ death benefit and $\$ 20$ weekly indemnity, annual premium, $\$ 57.60$

The maximum limit for engineers is $\$ 2,000$ death benefit and $\$ 20$ weekly indemnity; for firemen, $\$ \mathrm{I}, 500$ death benefit and $\$ \mathrm{I} 5$ weekly indemnity. The annual premium for weekly indemnity alnne is $\$ 10.80$ for $\$ 5$ or $\$ 43.20$ for $\$ 20$ weekly indemnity. The insured gives an order on the paymaster of the railroad company, according to previous contract, and the premium is taken out of the monthly pay. The figures of business in the year 1905 were: accident premiums received, $\$ 818,973$, and losses paid, 384,733 ; health premiums, $\$ 102,757$, losses, $\$ 40,97$ I. The statement does not show how many were wage-earners.

The Continental Casualty Company of Chicago does a large business in accident insurance. In the year 1905 it collected from wage-earners in premiums $\$ 1,675,000$; of this sum about 
$\$ 500,000$ was collected upon the industrial or "one-dollar-permonth plan." This company has already paid out to wage-earners for death benefits, sickness, and accident indemnities over $\$ 5,6$ I 5,000 .

Experiments are tried with various forms of sickness insurance and provision for invalidism. In discussing the burial benefit companies ("industrial insurance") we have seen that after an effort to unite sickness insurance with their business they abandoned the attempt, although the assessment companies studied by Forman in Washington still offer sick benefits in some policies. The chief difficulty in the experience of the most important companies was that there was no check on malingering and the cost was too high. Their officers thought that only in moderately small groups of fraternal societies would sickness insurance be practicable. The Health Insurance Company of Philadelphia and several companies in Massachusetts attempted to furnish sickness insurance about 1847 . The Philadelphia company started with a capital of $\$ 100,000$ in 1848 , used the tables of the English Friendly Societies as a basis of calculation, and charged from $\$ 5.25$ to $\$ 6.25$ for a weekly indemnity to cover loss from any kind of disease. Although commissions of agents were then very much lower than they could be now all these experiments ended in failure. Similar experiments and attempts in various parts of the country came to the same inglorious end; but so great is the need of such insurance, so disastrous the effects of being without protection, it was inevitable that the experiment should be revived in some form. About I896 some company introduced as a "rider" to an accident insurance policy an agreement, for a consideration, to pay indemnity in case of six zymotic diseases. This bait for accident insurance proved so attractive to customers that about 1899 several companies extended the list of diseases to ten or twelve, at a premium rate of $\$ 2$ for each $\$ 5$ of weekly indemnity, and under stress of competition among accident insurance companies the list was still further enlarged until about thirty or more were covered. Experience taught the companies, for a rather high tuition fee, that some of them had not charged enough for certain diseases to cover their risk 
and that the attempt to distinguish the nature of the sickness added to the confusion and cost attending adjustment of claims. To meet this situation a so-called General Disability Policy was introduced.

There can be no doubt that a policy covering any sickness originating in an individual after the beginning of his policy will afford less cause for misunderstanding and disagreement between the company and its policyholders, and that physicians will be less frequently called upon to stretch their consciences in diagnoses for the purpose of assisting their patients, than has been the case with the restricted sickness policy, and since many of the companies have taken up sickness insurance as an adjunct to accident insurance, merely as a means of holding their accident insurance against the aggressions of competing companies, any plan likely to secure this result with a minimum of friction and misunderstanding between the company and the assured would seem to commend itself to the underwriter if the cost does not prove to be too much of a tax upon the business which it is intended to protect. $^{\circ}$

Another suggestion has been made to diminish the cost of sickness insurance by connecting it with other kinds of insurance. It is evident that if one fee for soliciting and adjusting claims could be made to cover all forms of insurance desired, the sum of cost would be reduced, especially if the fee for solicitation did not have to be paid over each year. At present the company retains the right to stop protection at the end of any year or to increase the rate with age until it becomes prohibitive. Furthermore the benefit is limited to a relatively brief period, usually twenty-six weeks, while the need is for indemnity as long as sickness lasts. Mr. Dawson recommends that sickness insurance be joined with life insurance, for thus

it is possible to furnish at a much lower cost, because of lower expense in the payment of commissions, indemnity for the whole course of the disability, renewable without increase of premiums and at the option of the insured. Abundant statistics upon which to base these rates are now obtainable. ${ }^{7}$

Information from the mining region of the western states is difficult to secure, and therefore the statement of the superintend-

${ }^{6}$ R. S. Keelor, M.D., American Experience with Invalidity, I904.

${ }^{7}$ The Business of Life Insurance, p. 244. 
ent of social welfare of the Colorado Fuel and Iron Company, Pueblo, Colorado, is welcome.

This company provides medical attendance and hospital care for its employees and their families as long as they are sick, for which the men pay \$I per month. Privileges of the hospital, however, are extended only to the employees and not to their families, but a special rate is given to members of employees' families. All medical attendance outside of the hospital, including medicines, is furnished both to the men and to their families. Some years ago this company attempted to insure its miners against accidents, but the plan was not successful and so the plan at present followed by this and all other mining corporations in Colorado is to permit the agents of reliable insurance companies to go into the mines and solicit, the company guaranteing the agents the amount of premium which is then deducted from the men's payroll. The foreign miners also have a number of sick benefit societies, but they do not play a very prominent part in the matter of insurance, as they are usually small organizations. ${ }^{8}$

One point deserves special mention in connection with the assertion that compuslory insurance would be impossible unless all states introduced it at the same time, since the manufacturers of the state having compulsory insurance would have to carry heavier premiums than the managers in states which have not such laws. A part answer to this argument is found in the fact that already the cost of accidents must be borne in gifts, taxes for poor relief, and various schemes to which employers contribute for the relief of disabled men; and further, it may be claimed that insurance so greatly increases the contentment, steadiness, and efficiency of the insured workmen that premiums are largely returned in an equivalent of some kind. It may be added that if compulsory insurance were introduced in one state its advantages would soon be seen to be so great that public sentiment, reinforced by trade unions, would speedily make the law general in all industrial states. Still further it is precisely those states, as Massachusetts and New York, which lead in social legislation which retain the first rank as industrial states. To all this we may add certain facts furnished by casualty companies which tend to diminish the fears of timid capitalists that compulsory insurance would place them at a disadvantage as compared with the employers of other states; the fact being that

${ }^{8}$ Letter of Dr. R. W. Corwin. 
already, in consequence of the differences of court interpretations and legislation, the cost of employers' protection varies greatly in different states, without any of the dreadful things happening which are feared. Thus if we take the cost of liability insurance for the whole country as one, on the average, the cost for several states would be relatively as follows :

The figures are stated on a basis of a loss cost of I for the United States as a whole. If then the relative loss cost of a state is given as I.2, the meaning is that the loss cost in that state is twenty per cent. (20 per cent.) greater than for the United States as a whole. A relative loss of 2 designates a loss cost twice as great as that for the United States as a whole. A relative loss cost of .80 designates a loss cost eighty per cent. (8o per cent.) of that for the United States as a whole.

The loss cost of one state relatively to another may be ascertained by taking the ratio of their relative loss costs. Thus, the loss cost in Tennessee is four times as great as the loss cost in

TABLE OF RELATIVE LOSS COSTS TO EMPLOYERS IN THE SEVERAL STATES BY REASON OF THE LIABILITY IMPOSED BY LAW UPON THEM FOR DAMAGES ON ACCOUNT OF BODILY INJURIES OR DEATH ACCIDENTALLY SUFFERED BY THEIR EMPLOYEES ${ }^{1}$

\section{Table of States Listed Alphabetically}

\begin{tabular}{|c|c|c|}
\hline labama & Louisiana & \\
\hline rizona Territory ...2.00 & Maine $\ldots \ldots \ldots \ldots$ r.oo & Oklahoma Territory \\
\hline rkansas $\ldots \ldots \ldots$. . . 33 & Maryland $\quad \ldots . \ldots \ldots \cdot 70$ & Oregon $\ldots \ldots \ldots \ldots$ \\
\hline lifornia...$\ldots \ldots$ r.oo & Massachusetts ...... r.oo & ssylvania \\
\hline olorado $\quad \ldots . \ldots . . .2 .00$ & Michigan $\quad . . . \ldots .6 .60$ & Rhode Island \\
\hline Connecticut $\ldots \ldots .6$.6о & Minnesota .......... I.33 & South Carolina. \\
\hline Delaware .... & Mississippi $\quad \ldots \ldots \ldots .80$ & South Dakota \\
\hline District of Columbia. 90 & Missouri $\ldots \ldots \ldots \ldots$ r.33 & Tennessee ... \\
\hline lorida $\ldots \ldots \ldots \ldots$.6 & Montana & Texas \\
\hline ia $\quad \ldots \ldots \ldots \ldots$ r.2o & Nebrask & Utah \\
\hline $\begin{array}{l}\ldots \ldots \ldots \ldots \ldots 2.00 \\
\ldots \ldots \ldots \ldots \ldots r .33\end{array}$ & $\begin{array}{l}\text { New Hampshire .... } \\
\text { New Jeoo } \\
\text { Nersey ........ }\end{array}$ & Vermont \\
\hline ...1.20 & New Mexico Ty. ...2.00 & \\
\hline erritory $\ldots 2.00$ & Nevada & Washington · \\
\hline$\ldots \ldots \ldots \ldots$ r.33 & New York ........ & West Virginia \\
\hline$\ldots \ldots \ldots 2.00$ & North Carolina .....r.2o & onsin \\
\hline Kentr & North Dakota .. & Tyoming \\
\hline
\end{tabular}

${ }^{1}$ From the Monthly Bulletin of the Fidelity and Casualty Company. 
Pennsylvania, the relative loss cost of Tennessee being 2, that of Pennsylvania being 0.50 , and the ratio $2 \div 4=0.50$.

The differences between the states are due to differences in the law and in the judicial decisions interpreting the law.

The above table is founded in the main on combined experience of the companies composing the Liability Conference, an association of companies engaged in the business of liability insurance. Where changes in the law have occurred subsequent to the period embraced by the experience, comparative studies of the laws have been made and corrections made in the table accordingly. It is believed that the table represents accurately the relative costs today.

It is worth while to consider the probable part which the casualty companies will play in the immediate future in relation to the development of accident and sickness insurance, especially if permissive or compulsory laws should be passed in any of the states. This matter has already been seriously considered. It is well known that in England under the Compensation Act and recently in France under a compulsory-insurance law, the private accident companies have done a thriving business in assuming the legal obligations of the employers. In France the mutual insurance associations or syndicates, and even the government itself, through a central fund, are competitors of the private companies, and yet the latter hold their own and contribute very substantially to the promotion of the purposes of the law. Nor are we entirely without experience in the United States, for the workmen's collective policies contain suggestions of a method which may be greatly extended if legal pressure or even encouragment were to make it to the interest of large bodies of employers and wage-earners to unite in securing protection. Already under the collective policies the expenses of solicitation have been reduced to a minimum, since the entire body of employees is included at a stroke under a contract which also lowers the cost of payments of premiums by the simple process of deducting them from the wages. Uncertainty in regard to the indemnity would be reduced by legal definition of obligation and by simpler judicial organization for the adjustment of disputed claims. If 
the employers could be released from liability under existing laws they could then have at their disposal a large fund which they are now compelled to expend on casualty companies and lawyers to protect themselves against suits for negligence; and the insurance companies would then become insurers of the working-men rather than their sworn antagonists. 